\title{
Quasilinear transport modelling at low magnetic shear
}

J. Citrin, ${ }^{1, \text { a) }}$ C. Bourdelle, ${ }^{2}$ P. Cottier, ${ }^{2}$ D.F. Escande, ${ }^{3}$ Ö.D. Gürcan, ${ }^{4}$ D.R. Hatch, ${ }^{5}$

G.M.D. Hogeweij, ${ }^{1}$ F. Jenko, ${ }^{5}$ and M.J. Pueschel ${ }^{5}$

1) FOM Institute DIFFER - Dutch Institute for Fundamental Energy Research, Association EURATOM-FOM, Nieuwegein, The Netherlands

${ }^{2)}$ CEA, IRFM, F-13108 Saint Paul Lez Durance, France

3) UMR 6633 CNRS-Université de Provence, Marseille, France

4) Laboratoire de Physique des Plasmas, Ecole Polytechnique, CNRS, 91128 Palaiseau Cedex, France

5) Max-Planck-Institut für Plasmaphysik, EURATOM Association, D-85748 Garching, Germany

Accurate and computationally inexpensive transport models are vital for routine and robust predictions of tokamak turbulent transport. To this end, the QuaLiKiz [C. Bourdelle et al., Phys. Plasmas 14, 112501 (2007)] quasilinear gyrokinetic transport model has been recently developed. QuaLiKiz flux predictions have been validated by non-linear simulations over a wide range in parameter space. However, a discrepancy is found at low magnetic shear, where the quasilinear fluxes are significantly larger than the non-linear predictions. This discrepancy is found to stem from two distinct sources: the turbulence correlation length in the mixing length rule and an increase in the ratio between the quasilinear and non-linear transport weights, correlated with increased non-linear frequency broadening. Significantly closer agreement between the quasilinear and non-linear predictions is achieved through the development of an improved mixing length rule, whose assumptions are validated by non-linear simulations.

PACS numbers: 52.25.Fi, 52.30.Gz, 52.35.Ra, 52.55.Fa, 52.65.Tt, 52.65.-y

\footnotetext{
a) Electronic mail: J.Citrin@differ.nl
} 


\section{INTRODUCTION}

The availability and accuracy of computationally inexpensive first-principle physics based transport codes is vital for both interpretation of tokamak experiments and extrapolation to future devices. With this goal in mind, the QuaLiKiz quasi-linear transport model has been developed ${ }^{1}$. Particle and heat flux calculation time in QuaLiKiz is $\sim 50000$ less than in local non-linear codes. Fluxes computed by QuaLiKiz have been validated by non-linear $\mathrm{GYRO}^{2,3}$ simulations over a wide range of parameter space ${ }^{4}$. However, significant discrepancies between QuaLiKiz and non-linear simulations have been observed at low magnetic shear.

The successful prediction of transport in the low magnetic shear regime is important for the interpretation of advanced tokamak scenarios, as has been recently seen in JET hybrid scenarios at combined low magnetic shear and high rotational shear ${ }^{5}$. Low magnetic shear regions are also expected during ramp-up and ramp-down phases. For extrapolation to ITER, transport at low magnetic shear is important for all scenarios. This is due to the fact that in ITER, the sawtooth period is expected to be an order of magnitude greater than the confinement time ${ }^{6}$. Therefore even in the ITER reference H-mode scenario, low magnetic shear transport within the inversion radius between sawtooth crashes will be critical for determining the average fusion power.

The primary motivation of this work is thus to understand the physics behind the low magnetic shear discrepancy between the QuaLiKiz model as formulated in Casati et $a l^{4}$, and non-linear simulations. To this end, the assumptions made in the QuaLiKiz model are systematically examined at low magnetic shear. Both linear and non-linear simulations with the gyrokinetic code $\mathrm{GENE}^{7}$ have been used extensively for comparisons and assumption validation.

The structure of the paper is as follows. In section II the QuaLiKiz model is reviewed and the discrepancy compared to non-linear simulations at low magnetic shear is introduced. In section III, a review of basic understandings of the magnetic shear impact on linear modes is presented. QuaLiKiz and GENE linear growth rates are compared at low magnetic shear, and the differences between them are not sufficient to explain the flux discrepancy. In section IV, QuaLiKiz assumptions on non-linear effects are explored. The validity of the quasi-linear approximation itself with regard to the ordering of the non-linear characteristic 
times (Kubo numbers) is examined, and is found to still hold at low magnetic shear. The delicate choice of electrostatic potential saturation level, through the mixing length rule and frequency broadening, is reevaluated at low magnetic shear. The assumptions behind the saturated potential formulation have been reexamined in great detail with the aid of 16 nonlinear GEnE simulations at various values of magnetic shear, q-profile and driving gradient lengths. Partial isotropization of turbulent eddies, radial correlation length dependence on the magnetic shear, and increased resonance broadening at low magnetic shear are all observed. These observations are taken into account in the quasilinear model, and agreement with the non-linear magnetic shear scans is much improved. Conclusions are discussed in section $\mathrm{V}$.

A number of more technical aspects have been summarized in appendixes. In appendix A the analytical fluid limit applied in the QuaLiKiz Gaussian eigenmode ansatz is reviewed. In appendix B the locality assumption is examined at low magnetic shear, and found to hold for $|s|>0.1$. In appendix $\mathrm{C}$, the validity of the QuaLiKiz eigenmode ansatz is examined at low magnetic shear, through comparisons with self-consistent mode structure calculations from linear-Gene. This QuaLiKiz assumption was also found to hold for $|s|>0.1$. Recent improvements in the QuaLiKiz linear solver are summarized in the remaining appendixes: treatment of the bounce average and bounce frequency resonance in the trapped particle functional (appendix D), mode width calculation in $k_{\theta} \rho_{s} \gg 1$ ETG relevant wavenumbers (appendix E), and mode width calculation in the vicinity of $s \sim 0.5$ (appendix F).

Many of the simulations carried out in this work refer to 'GA-standard case' parameters. For reference, we list these parameters here: $r / a=0.5, R / L_{T i, e}=9, R / L_{n}=3, q=2$, $T_{i} / T_{e}=1, s=1, \beta=0, \nu^{*}=0$.

Finally, we clarify here the wavenumber notation used in this work. $k_{y}$ is the wavenumber, normalized to $1 / \rho_{s}$, corresponding to the binormal coordinate $y$ in the GENE field-aligned coordinate system. In QuaLiKiz, $k_{\theta}$ is used, which corresponds to the wavenumber in the poloidal direction. For all parameters studied in this work, $k_{y}$ differs from $k_{\theta} \rho_{s}$ by at most $2 \%$. Thus for convenience we will not differentiate between the two, and henceforth always use $k_{y}$ in reference to all simulation results. 


\section{REVIEW OF THE QUALIKIZ MODEL}

QuaLiKiz is based on the electrostatic eigenvalue gyrokinetic code Kinezero ${ }^{8}$, which includes two ion species and both passing and trapped electrons. For the minimization of computational expense - critical for integrated modelling applications - the electrostatic potential shape is not calculated self-consistently. Rather, an analytical solution obtained in the fluid limit is taken and the strong ballooning representation limit applied. A Krook-like collision operator accounting for the impact of collisions on the trapped electrons has also been included ${ }^{9}$. Shifted circle $(s-\alpha)$ geometry is assumed for equilibrium.

The linear response calculated by Kinezero has been incorporated into the gyrokinetic quasilinear expressions for the particle and heat fluxes, resulting in the QuaLiKiz transport model $^{1}$. From henceforth we will also refer to the eigenvalue solver as part of 'the QuaLiKiz model'. The fluxes can be schematically decomposed into contributions of the linear response and the saturated electrostatic potential:

$$
\Gamma, Q_{i}, Q_{e}=\sum_{k_{\theta}, \omega, \omega_{k j}} \operatorname{Im}\left(R_{l i n}^{\Gamma, Q_{i}, Q_{e}}\left(k_{\theta}, \omega, \omega_{k j}\right)\right)\left|\delta \phi\left(k_{\theta}, \omega, \omega_{k j}\right)\right|^{2}
$$

Where $R_{\text {lin }}^{\Gamma, Q_{i}, Q_{e}}$ is the linear response for the density, ion temperature, and electron temperature fluctuations respectively, $\delta \phi$ the saturated electrostatic potential, $\omega$ the fluctuation frequency, and $\omega_{k j}$ the eigenfrequency of the unstable mode with poloidal wavenumber $k_{\theta}=n q / r$. Multiple instabilities may coexist with the same $k_{\theta}$, and are discriminated by the index $j$. The contribution of each unstable mode (also named 'branches') to the fluxes are summed over. The linear response has been extensively validated by comparison with non-linear simulations ${ }^{4,10}$.

The model for the saturated electrostatic potential - a critical ingredient in evaluating the turbulent fluxes - consists of a mixing length rule weighted by a k-space spectral function validated against both non-linear simulations and experimental observations ${ }^{4,11}$. The saturated potential frequency spectral shape is taken as a Lorentzian, with a width equal to the linear growth rate. This assumption has been shown to agree with non-linear simulations and experimental observations at transport relevant wavelengths ${ }^{10}$. Finally, the QuaLiKiz predicted fluxes are normalized by a single, constant, fitting coefficient, set such that the ion heat flux predicted by QuaLiKiz for the GA-standard case agrees with GYRO predictions.

The mixing length rule as previously applied in QuaLiKiz is now presented. We rede- 
fine for convenience the saturated electrostatic potential for each mode as $\left|\delta \phi_{k, j}\right|^{2}$, where $k$ is the wavenumber index and $j$ the branch index. We assume $\left|\delta \phi_{k, j}\right|^{2}=S_{k j} L_{j}$, where $S_{k j}$ is a branch dependent k-space spectral function shape, and $L_{j}$ is the maximum mixing length amplitude for branch $j: L_{j} \equiv\left|\frac{\gamma_{k, j}}{\left\langle k_{\perp}^{2}\right\rangle}\right|_{\max }$. The maximum is taken over the k-spectrum. The $k_{\theta}$ corresponding to the maximum is denoted $\left.k_{\theta}\right|_{\max }$, and represents the peak of the flux spectrum defined by $S_{k j}$, designed to reproduce the downshifted non-linear flux spectrum $^{12}$. $S_{k j}$ is taken such that $S_{k j} \propto\left(k_{\theta}\right)^{3}$ for $k_{\theta}<\left.k_{\theta}\right|_{\max }$, and $S_{k j} \propto\left(k_{\theta}\right)^{-3}$ for $k_{\theta}>\left.k_{\theta}\right|_{\max }$. $\left\langle k_{\perp}^{2}\right\rangle=k_{\theta}^{2}\left(1+(s-\alpha)^{2}\left\langle\theta^{2}\right\rangle\right)$, where \langle\rangle denotes a poloidal average. This average over the ballooning structure, when integrating over the form imposed by the fluid Gaussian eigenmode assumption, is given by $\left\langle\theta^{2}\right\rangle=\frac{2 d^{2} \Gamma(3 / 4)}{w^{2} \Gamma(1 / 4)}$. $\mathrm{d}$ is the distance between rational surfaces and $w$ is the mode Gaussian width. This choice of k-space spectrum is in agreement with nonlinear simulations, and observations ${ }^{4,11}$.

Fluxes computed by QuaLiKiz have been compared with GYRO non-linear simulations, and agreement for both heat and particle fluxes have been obtained in $R / L_{T}, Z_{\text {eff }}, T_{i} / T_{e}$ and collisionality scans around GA-standard case parameters ${ }^{4}$. Extensive GYRo non-linear simulations consisting of q-profile and magnetic shear scans have previously been carried out $^{13}$. In figure 1 , the $R / L_{T}$ scan comparison is reproduced, and q-profile and magnetic shear scans around the GA standard case have been added. The q-profile scan also shows agreement between the predictions. However, significant discrepancies between QuaLiKiz and GYRO are evident in the magnetic shear scan, in the region $|s|<0.5$. Note that $\alpha=0$ in all these calculations. We now set out to uncover the sources of this discrepancy.

\section{MAGNETIC SHEAR IMPACT ON LINEAR PHYSICS}

In this section we review linear growth rate dependence on magnetic shear, and compare QuaLiKiz and linear-GENE growth rate calculations. Both the role of the magnetic shear on the $R / L_{T i}$ thresholds of ITG instabilities and on $\gamma$-stiffness is discussed. $\gamma$-stiffness is defined as the rate of change of the growth rate as a function of the driving gradient lengths. In section III A analytical and computational results for the ITG instability threshold are briefly reviewed. While in the fluid limit we expect higher growth rates for higher $s / q$ (see appendix A), the linear thresholds of the ITG modes are in fact expected to decrease with $s / q$. This points to reduced $\gamma$-stiffness at low magnetic shear. Particularly, the toroidal 

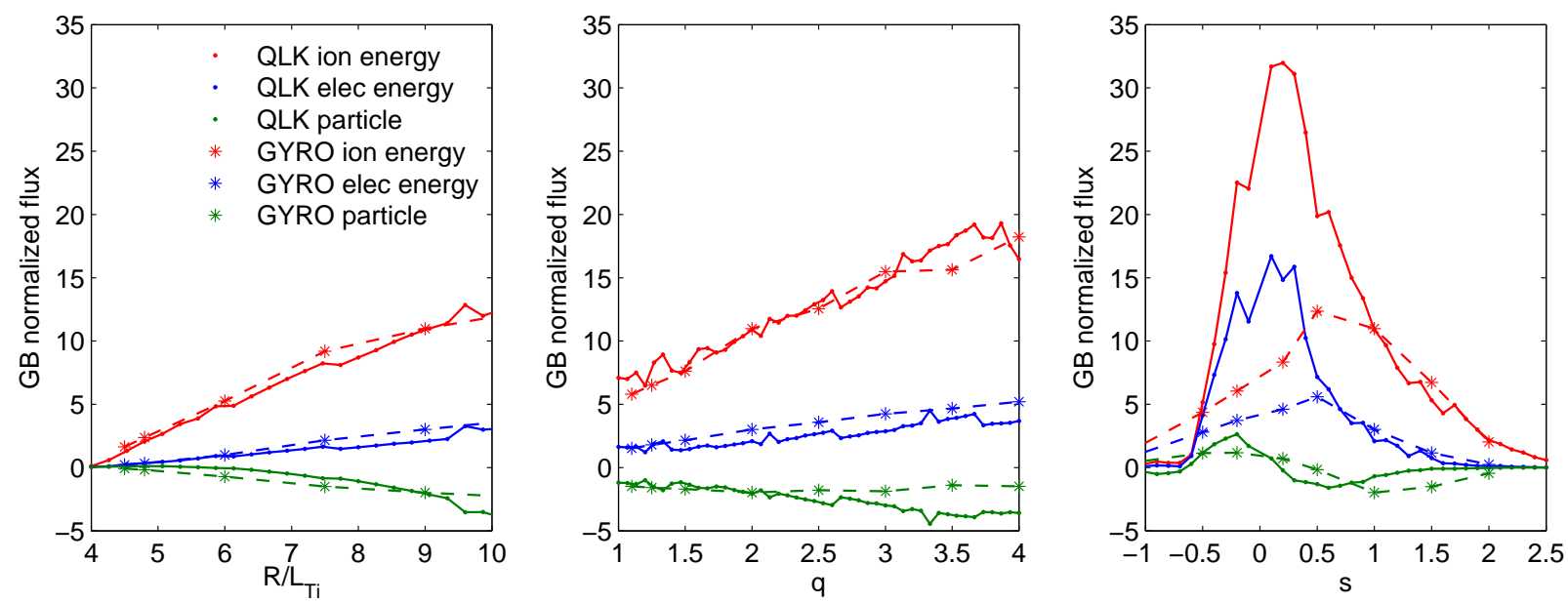

FIG. 1. Ion and electron flux, compared between GYRO and QuaLiKiz for 3 scans around the GASTD case: $R / L_{T}$ (left panel), $q$ (central panel), and $s$ (right panel). This comparison is with the previous mixing length formulation in QuaLiKiz as presented in Casati et al ${ }^{4}$.

ITG branch modes at low $k_{y} s$ are stabilized. The analytical linear results are corroborated by computations with both linear-Gene and QuaLiKiz. The agreement between dominant branch growth rate calculations in linear-GENE and QuaLiKiz is satisfactory at low magnetic shear. This leads to the conclusion that the reason for the discrepancy between the quasilinear and non-linear fluxes at low magnetic shear lies in the non-linear physics.

\section{A. Linear thresholds from kinetic theory}

In this section we briefly review previous results of kinetic theory regarding magnetic shear dependences on threshold, for ITG turbulence. For the $\eta_{i}$ ITG mode $\left(\eta_{i} \equiv d \ln T_{i} / d \ln n\right)$, the $R / L_{T i}$ threshold has been calculated in the short-wavelength, flat density limit $\left(k_{\theta} \rho_{s} s \approx\left(L_{T} / R\right)^{1 / 4}\right.$, $\left.R / L_{n}<2\left(1+T_{i} / T_{e}\right)\right)$, as follows ${ }^{15}$ :

$$
\left(R / L_{T i}\right)_{c} \approx \frac{4}{3}\left(1+T_{i} / T_{e}\right)\left(1+2 \frac{s}{q}\right)
$$

Increasing the density gradient is predicted to lead to an increased $R / L_{T i}$ threshold. However, when including trapped electrons the increased density gradient can destabilize the TEM branch, nullifying the turbulence stabilization ${ }^{16}$.

A study of the (s,q) impact on the linear $R / L_{T}$ threshold for ITG/TEM modes in Kinezero (and thus in QuaLiKiz) have previously been carried out with fixed $T_{i} / T_{e}=Z_{\text {eff }}=1, \alpha=0$. 
and in the flat density limit ${ }^{19}$. The parameterization of the critical temperature gradient lengths, $\left(R / L_{T}\right)_{c}$, was as follows for ITG-like modes: $1.1+1.4 s+1.9 s / q$ for $s>0$, and $0.9+1.6|s|+9.9|s| / q$ for $s<0$. This mixed $s$ and $s / q$ dependency share the same qualitative dependencies as in the analytical limits above. The asymmetry with the sign of $s$ is due to the stabilization of the toroidal branch for negative shear. An extensive study of $T_{i} / T_{e}$ dependence in QuaLiKiz was carried out by Casati et $a l^{20}$.

Finally, comprehensive linear gyrokinetic simulations with GS2 have led to a parameterization of ETG linear thresholds, isomorphic with ITG linear thresholds (assuming adiabatic electrons), for which it can be written as follows ${ }^{21}$ :

$$
\left(R / L_{T i}\right)_{c}=\max \left\{\left(1+T_{i} / T_{e}\right)(1.33+1.91 s / q)(1-1.5 \epsilon) \times[1+0.3 \epsilon(d \kappa / d \epsilon),], 0.8 R / L_{n}\right\}
$$

where $\epsilon=r / R$ and $\kappa$ is the elongation. In the circular cross-section limit, this formula is very similar to the analytical formula, equation 2.

Growth rate contour plots calculated by QuaLiKiz are shown in figure 2. The growth rates correspond to the most unstable branch in GA-standard $R / L_{T}$ and magnetic shear scans (where $R / L_{T i}=R / L_{T e}$ ). In the left panel, the maximum growth rate for the spectrum $k_{y}<0.8$ is displayed. In the right panel, the growth rates for $k_{y}=0.2$ are displayed. In both plots, the increased instability threshold for high magnetic shear and negative magnetic shear is clearly visible. The residual modes at low $R / L_{T}$ at low magnetic shear are modes in the electron diamagnetic direction due to the finite $R / L_{n}$ in the GA-standard case.

An interesting detail to note in the low $k_{y}$ case (right panel) of figure 2 is reduced $\gamma$ stiffness at low magnetic shear. This is evidenced by the significantly decreased gradient with respect to $R / L_{T}$ at $s<0.2$. A possible explanation for this decreased $\gamma$-stiffness is that the long-wavelength limit is reached, defined by $k_{y} s \ll\left(L_{T i} / R\right)^{1 / 4}$. In this limit, the toroidal branch is stabilized ${ }^{22}$. This is due to the growth of the eigenfunction envelope width along the magnetic field, given by $\theta \approx s^{-1 / 2} \epsilon_{T}^{1 / 4}\left(k_{\theta} \rho_{i}\right)^{-1}$. A broad envelope leads to decreased interaction with the bad curvature region, stabilizing the toroidal branch. In the QuaLiKiz Gaussian eigenmode approximation the envelope width is proportional to $d / w$, which increases at low $k_{y}$, and also increases at low magnetic shear as seen in figure 5 in Romanelli et $a l^{9}$. Decreased linear growth rates at low $k_{y} s$ may lead to preferential quenching of long wavelength turbulence by zonal flows at low magnetic shear. Since these 
long wavelengths dominate the transport, this can play a major role in the determination of the fluxes.
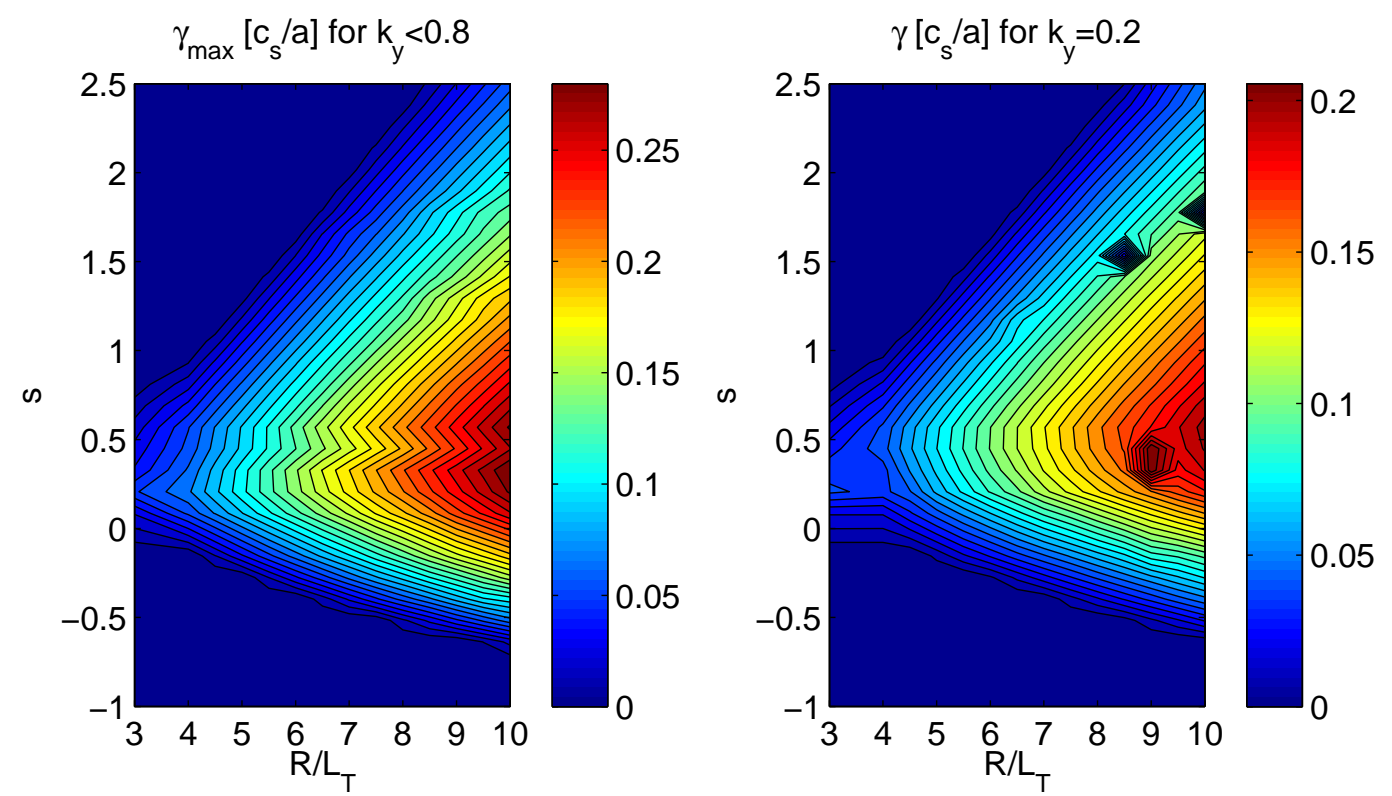

FIG. 2. Linear growth rates for the most unstable branch calculated by QuaLiKiz in a GA-STD $R / L_{T}$ scan, where $R / L_{T i}=R / L_{T e}$. In the left panel the maximum growth rate for the spectrum $k_{y}<0.8$ is shown. In the right panel, the growth rate for $k_{y}=0.2$ is shown.

\section{B. Comparison of QuaLiKiz and linear-GENE growth rates}

Growth rates of the dominant branch calculated by QuaLiKiz and GENE are compared in this section. In figure 3 results of a $R / L_{T}$ scan are displayed (where $R / L_{T i}=R / L_{T e}$ throughout the scan), around the GA-standard case, at $k_{y}=0.15,0.3$ and $s=0.1,1$. We can see that even though the $R / L_{T}$ thresholds are lower for the lower shear cases, the $\gamma$ stiffness is also lower for the lower shear case, leading to a crossover of the high-shear and low-shear $\gamma$ curves at a $R / L_{T}$ position dependent on $k_{y}$. At low $k_{y}$, this crossing point is much closer to the threshold, at experimentally relevant $R / L_{T}$. Note that this effect is predicted by both GENE and QuaLiKiz, in spite of the Gaussian eigenmode ansatz made in QuaLiKiz. Indeed, it is shown in Appendix $\mathrm{C}$ that this ansatz is valid down to $s=0.1$, $k_{y}=0.15$.

We also show in figure 4 a comparison between linear-GENE and QuaLiKiz of the growth 

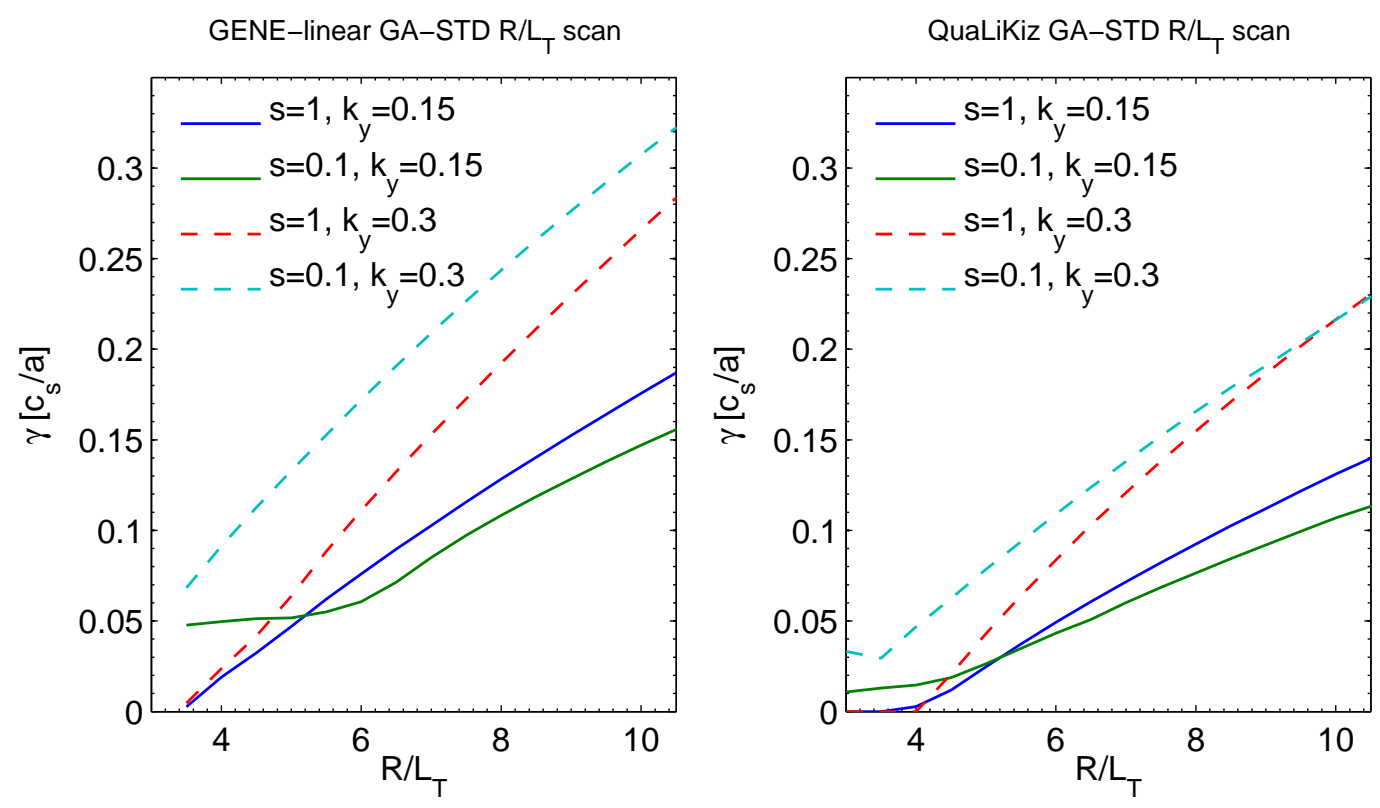

FIG. 3. Linear growth rates calculated for the GA-STD kinetic electron case in a $R / L_{T}$ scan at various $k_{y}$ and magnetic shear values by both linear-Gene (left panel) and QuaLiKiz (right panel)

rate calculation for a GA-standard magnetic shear scan at $k_{y}=0.3$. The QuaLiKiz predictions agree with linear-GENE to within $\sim 30 \%$ even at low magnetic shear. However, at negative magnetic shear QuaLiKiz severely underpredicts the growth rates, due to an underestimation of the slab ITG branch growth rate. This may be due to the strong ballooning representation and prescribed eigenmode assumptions. The resolution of this issue is left for future work.

In summary, while the ITG threshold decreases at low magnetic shear, the $\gamma$-stiffness is also reduced at lower magnetic shear. At low $k_{y}$ this effect is increased. This is reproduced both by linear-Gene and QuaLiKiz. Furthermore, dominant branch linear-Gene and QuaLiKiz calculations agree to within $\sim 30 \%$ in the parameter ranges studied, in spite of the QuaLiKiz Gaussian eigenmode ansatz. Therefore a miscalculation of the dominant branch growth rate cannot be responsible for the low magnetic shear flux discrepancy between QuaLiKiz and non-linear models, where the QuaLiKiz flux is significantly higher as seen in figure 1 . The reduced growth rates at combined low magnetic shear and low $k_{y}$ is an important point, since this could have ramifications for setting the turbulence correlation length. This is explored further in the modification of the mixing length rule in section IV C. 


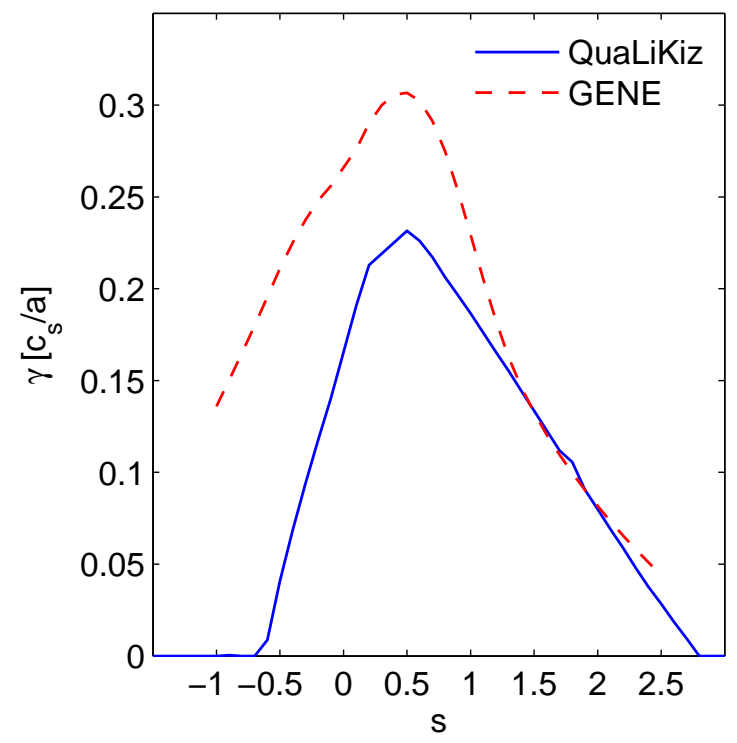

FIG. 4. Linear growth rates calculated for a magnetic shear scan around the GA-standard case by both QuaLiKiz (solid lines) and linear-GENE (dashed lines) for $k_{y}=0.3$

\section{NON-LINEAR EFFECTS AT LOW MAGNETIC SHEAR}

In this section we explore the non-linear physics which may lead to the lack of validity of the previous QuaLiKiz quasilinear computed fluxes at low magnetic shear. As described in section I, the quasilinear flux can be decomposed into two parts: the linear response and the saturated electrostatic potential. This description is justified if the particles undergo stochastic linearized trajectories. This assumption, central to quasilinear theory, can be verified by examining the ordering of the turbulence autocorrelation time compared to the eddy turnover time, as expressed through the Kubo number. This verification was done here for low magnetic shear. Furthermore, the QuaLiKiz mixing length model for the non-linear saturated potential was also reexamined at low magnetic shear. This includes the model for $k_{\perp}$, the evaluation of the $k_{y}$ maximum of the non-linear flux spectrum, and the frequency broadening assumption. These assumptions were extensively validated previously for a wide range of parameters ${ }^{4}$, but not for magnetic shear scans. We extend this validation towards low magnetic shear.

A set of dedicated GENE non-linear runs were carried out to test and validate the QuaLiKiz assumptions. These non-linear runs are introduced in section IV A. In section IV B the evaluation of the Kubo numbers in the non-linear simulations are discussed. In section IV C 
TABLE I. Input parameters for the GENE magnetic shear scans

\begin{tabular}{c|c|c|c|c|c} 
Set name & $R / L_{T i}$ & $R / L_{T e}$ & $R / L_{n}$ & $T_{i} / T_{e}$ & $\mathrm{q}$ \\
\hline $\mathrm{A}$ (GASTD) & 9 & 9 & 3 & 1 & 2 \\
\hline $\mathrm{B}$ & 7.6 & 6.7 & 4 & 1.4 & 1.4 \\
\hline $\mathrm{C}$ & 6.3 & 5.5 & 3.3 & 1.4 & 1.4 \\
\hline $\mathrm{D}$ & 7.6 & 6.7 & 4 & 1.4 & 1
\end{tabular}

the mixing length model for the saturated potential is reexamined, and modified following comparisons with the non-linear simulations. Finally, predicted fluxes from both the modified QuaLiKiz and the set of non-linear GENE simulations are compared in section IVD.

\section{A. Description of GENE runs}

A total of 16 non-linear GENE runs were carried out. The runs are comprised of 4 sets of 4 -point magnetic shear scans at $s=-0.4,0.1,0.6,1$. The sets are comprised of the GAstandard case, and 3 further sets with lowered inverse gradient lengths and varying q-profile values, consistent with typical JET hybrid scenario parameters at mid-radius. This was done in order to widen the GENE-QuaLiKiz magnetic shear scan validation in q-profile parameter space, down towards $q=1$ which often coincides with low magnetic shear in experiments. Furthermore, the added data sets allow GENE-QuaLiKiz validation at lower levels of flux than the GA-standard case. Since the eventual goal is to apply QuaLiKiz as a transport model within the integrated modelling framework, validation at more experimentally relevant fluxes is critical. The parameters for these sets are summarized in table I. All GEnE simulations carried out were electrostatic, local, collisionless, with periodic radial boundary conditions, kinetic electrons (and real mass ratio), and with $s-\alpha$ geometry. Throughout the sets, $\alpha=0, r / a=0.5$, and $R_{0} / a=3$.

In all simulations, we used 16-point discretization in the parallel direction, 32 or 48-points in the parallel velocity directions (based on linear convergence studies), and 8 magnetic moments. For these parameters, non-linear convergence tests were carried out, and the heat flux sensitivities were negligible. For each simulation, extensive non-linear convergence studies were carried out in the number of toroidal modes, the perpendicular box sizes, and in the radial grid discretization. The box sizes in the perpendicular directions range from 
$\left[L_{x} / \rho_{s}, L_{y} / \rho_{s}\right]=[100,126]$ for the set A, $s=1$ case to up to $\left[L_{x} / \rho_{s}, L_{y} / \rho_{s}\right]=[333,210]$ for the more challenging $s=0.1$ cases. The number of toroidal modes $\left(n_{y}\right)$ employed ranged from 16 for the set A, $s=1$ case, to 32 for the $s=0.1$ cases. The $n_{y}=32$ cases correspond to $k_{y}(\max )=1.55$ or $k_{y}(\max )=0.93$, depending on the perpendicular box size. In general, the $s=0.1$ cases were more computationally intensive due to the higher radial box size needed to satisfy the parallel boundary condition, and also due to the higher number of toroidal modes necessary for convergence.

Low heat flux sensitivity was also found to the inclusion of collisions. However, it was found that the particle transport was highly sensitive to the velocity and parallel space resolutions, particularly when a particle inward flux dominates (as in the set A, $s=1$ case). This particle transport sensitivity was greatly decreased when including collisions. In general, verification of the QuaLiKiz particle transport predictions is also of great importance. However, it is preferable to carry out this verification vs non-linear runs when including collisionality. This is due both to the increased experimental relevance, and also the seemingly improved non-linear convergence properties of particle diffusivity in collisional runs. Due to the increased computational expense of collisional runs, a more rigorous validation of the particle fluxes when including collisionality is deferred to future work. Here we concentrate on the heat transport validation of the collisionless QuaLiKiz model at low magnetic shear, and maintain a caveat on the convergence of the non-linearly calculated particle fluxes in this work, particularly the inward fluxes. The discretization and box size choices, as well as the transport predictions of all 16 runs, are shown in table II.

\section{B. Kubo numbers}

The validity of the quasilinear assumption depends on whether test particles in the turbulent fields undergo stochastic linearized trajectories, allowing a diffusive random-walk process to be considered as opposed to field trapping. This can be quantified by the Kubo number $K^{23,24}$, the ratio between the Eulerian autocorrelation time, $\tau_{a c}$, of the fluctuating potential and the particle flight time, $\tau_{f} . \tau_{f}$ is defined as $\lambda_{x} /\left\langle v_{x}\right\rangle$, where $\lambda_{x}$ is the radial correlation decay length, and $\left\langle v_{x}\right\rangle$ the root mean square radial velocity of a test particle in the turbulent field. For $K<1$, the autocorrelation time of the turbulent field is shorter than the transit time of a test particle around a turbulent eddy, justifying the use of a random-walk diffusive 
TABLE II. Grid settings and results of converged GENE non-linear runs

\begin{tabular}{c|c|c|c|c|c|c|c|c|c|} 
Run & $n_{x}$ & $n_{y}$ & $k_{y} \min$ & $n_{z}$ & $n_{v}$ & $n_{w}$ & $L_{x}$ & Sim time $\left[R / c_{s}\right]$ & $\chi_{i} / \chi_{e} / D$ [GB units] \\
\hline A: s=1 & 140 & 16 & 0.05 & 16 & 32 & 8 & 100 & 950 & $39.64 / 9 /-0.75$ \\
A: s=0.6 & 140 & 16 & 0.05 & 16 & 48 & 8 & 140 & 960 & $39.94 / 13.21 / 0.26$ \\
A: s=0.1 & 256 & 24 & 0.05 & 16 & 48 & 8 & 200 & 560 & $21.84 / 11.51 / 2.57$ \\
A: s=-0.4 & 256 & 25 & 0.03 & 16 & 32 & 8 & 166 & 630 & $23.66 / 11.67 / 5.36$ \\
\hline \hline B: s=1 & 140 & 16 & 0.05 & 16 & 48 & 8 & 140 & 800 & $18.33 / 5.7 / 0.9$ \\
B: s=0.6 & 160 & 32 & 0.03 & 16 & 48 & 8 & 160 & 750 & $25.58 / 11.64 / 3.32$ \\
B: s=0.1 & 300 & 32 & 0.03 & 16 & 48 & 8 & 333 & 720 & $11.63 / 7.88 / 2.84$ \\
B: s=-0.4 & 160 & 32 & 0.03 & 16 & 48 & 8 & 160 & 640 & $13.37 / 9.62 / 3.86$ \\
\hline \hline C: s=1 & 140 & 16 & 0.05 & 16 & 48 & 8 & 140 & 1570 & $9.54 / 1.77 /-0.01$ \\
C: s=0.6 & 210 & 24 & 0.05 & 16 & 48 & 8 & 140 & 600 & $17.65 / 5.22 / 0.95$ \\
C: s=0.1 & 300 & 32 & 0.03 & 16 & 48 & 8 & 200 & 600 & $7.74 / 4.01 / 1.29$ \\
C: s=-0.4 & 240 & 24 & 0.05 & 16 & 48 & 8 & 160 & 610 & $7.32 / 4.89 / 1.9$ \\
\hline \hline D: s=1 & 200 & 32 & 0.05 & 16 & 48 & 8 & 200 & 610 & $8.94 / 2.25 / 0.05$ \\
D: s=0.6 & 240 & 32 & 0.05 & 16 & 48 & 8 & 160 & 385 & $15.69 / 6.08 / 1.46$ \\
D: s=0.1 & 300 & 32 & 0.05 & 16 & 48 & 8 & 200 & 560 & $9.18 / 5.42 / 1.87$ \\
D: s=-0.4 & 240 & 32 & 0.05 & 16 & 48 & 8 & 160 & 380 & $7.87 / 5.61 / 2.2$
\end{tabular}

model. This condition is however necessary but not sufficient. In addition, there must be enough randomness in the Hamiltonian described in the dynamics, e.g. when it includes many waves with random phases ${ }^{25}$. This condition though is well satisfied in the strong turbulence cases studied here. For a $R / L_{T}$ scan based on the GA-standard case, computed using Gyro, it was seen that $K<1^{4}$. This was done calculating the autocorrelation time as $\tau_{a c}=2 D /\left\langle\left|v_{x}\right|^{2}\right\rangle$, where $\mathrm{D}$ is the particle diffusivity, as carried out in ${ }^{26}$. This assumes a priori that the particle transport is due to a random-walk process in the oscillating field. In this work we calculate the Kubo number by a more direct method, by calculating the $\tau_{a c}$ directly from the fluctuating potential field from GENE non-linear simulations. The time correlation function is $C(\Delta t)=\langle\phi(x, y, t) \phi(x, y, t+\Delta t)\rangle /\left\langle\left|\phi^{2}\right|\right\rangle$, averaging over the $\mathrm{x}$ and $\mathrm{y}$ directions. The parallel coordinate is kept fixed at the low-field side. The correlation time is taken as the $1 /$ e time of this function. All $n=0$ modes are not included in the calculation, 
which thus considers only the flux inducing $n>0$ background drift-wave population. $\lambda_{x}$ is calculated as the $1 / e$ length of the function $C(\Delta x)=\langle\phi(x, 0, t) \phi(x+\Delta x, 0, t)\rangle /\left\langle\left|\phi^{2}\right|\right\rangle$.

$\left\langle v_{x}\right\rangle=\sqrt{\left\langle E_{y}^{2}\right\rangle} / B_{\text {ref }}$, with the same averaging procedure as $\tau_{a c}$ for consistency. $B_{\text {ref }}$ is the reference magnetic field used to normalize the GENE equations and variables.

We have calculated the Kubo numbers for the full set of GENE non-linear runs. The calculated correlation lengths, times and Kubo numbers can be found in table III. For all cases, $K<1$. We thus conclude that the quasilinear approximation indeed holds. Note furthermore that $K<1$ but still of order unity supports the application of the mixing length rule for setting the level of the saturated potential, where the implicit assumption is that the step length of the random walk process is the eddy size. While the quasilinear approximation itself may hold, the value of the saturated electrostatic potential cannot be determined by linear theory. This value is determined by a mixing length rule, whose validation at low magnetic shear is evaluated in the next section.

\section{Reevaluation of the QuaLiKiz saturated potential model}

The model for the saturated potential is comprised of three parts: the model for $k_{\perp}$, setting the maximum and spectral shape of the non-linear flux spectrum, and frequency broadening. We briefly recall here the previous model. The $k_{y}$ maximum of the flux spectrum is designated $\left.k_{y}\right|_{\max }$, and corresponds to the maximum of the mixing-length flux spectrum $\frac{\gamma}{k}_{\left\langle k_{\perp}^{2}\right\rangle}^{12,27}$, where:

$$
\left\langle k_{\perp}^{2}\right\rangle=k_{y}^{2}\left(1+(s-\alpha)^{2}\left\langle\theta^{2}\right\rangle\right)
$$

This formulation of $k_{\perp}$ calculates only the effective $k_{x}$ due to the shearing of the mode along the field line, and assumes that the dominant instability has intrinsic $k_{x}=0$.

The k-spectrum is $k_{y}^{-3}$ and $k_{y}^{3}$ above and below the flux spectrum maximum respectively. The frequency spectrum of the saturated potential is broadened by the linear growth rate as follows: $\phi_{k}(\omega) \propto \frac{\gamma_{k}}{\left(\omega-\omega_{k}\right)^{2}+\gamma_{k}^{2}}$. This model has been shown to lead to discrepancies both in the $k_{y}$ of the saturated potential peak and in the frequency spectrum width when compared to Gene non-linear results at low magnetic shear. The k-spectrum model is reviewed and an improved formulation for $k_{\perp}$ is proposed in section IV C1. The frequency spectrum broadening comparison and consequences of the discrepancy are addressed in section IV C 2 . 
TABLE III. Correlation times, lengths and Kubo numbers for the GENE non-linear runs. Units are Larmor radii for the lengths, and $R / c_{s}$ for the times

\begin{tabular}{c|c|c|c|c|} 
Run & $\sqrt{\left\langle E_{y}^{2}\right\rangle}$ & $\lambda_{x}$ & $t_{a c}$ & $\mathrm{~K}$ \\
\hline $\mathrm{A}: \mathrm{s}=1$ & 8.6 & 12.60 & 1.85 & 0.42 \\
$\mathrm{~A}: \mathrm{s}=0.6$ & 11.78 & 11.2 & 2.05 & 0.72 \\
$\mathrm{~A}: \mathrm{s}=0.1$ & 12.23 & 7.12 & 0.58 & 0.33 \\
$\mathrm{~A}: \mathrm{s}=-0.4$ & 9.66 & 6.94 & 1.85 & 0.86 \\
\hline \hline B: $\mathrm{s}=1$ & 8.7 & 12.70 & 2.14 & 0.49 \\
$\mathrm{~B}: \mathrm{s}=0.6$ & 10.8 & 10.50 & 1.74 & 0.60 \\
$\mathrm{~B}: \mathrm{s}=0.1$ & 8.59 & 6.90 & 1.09 & 0.45 \\
$\mathrm{~B}: \mathrm{s}=-0.4$ & 7.93 & 6.87 & 2.14 & 0.82 \\
\hline \hline C: $\mathrm{s}=1$ & 5.37 & 13.67 & 1.90 & 0.27 \\
$\mathrm{C}: \mathrm{s}=0.6$ & 8.48 & 10.92 & 3.44 & 0.89 \\
$\mathrm{C}: \mathrm{s}=0.1$ & 6.69 & 7.30 & 1.44 & 0.44 \\
$\mathrm{C}: \mathrm{s}=-0.4$ & 5.5 & 6.08 & 2.01 & 0.61 \\
\hline \hline D: $\mathrm{s}=1$ & 7.38 & 9.76 & 0.96 & 0.24 \\
$\mathrm{D}: \mathrm{s}=0.6$ & 9.23 & 8.41 & 1.38 & 0.50 \\
$\mathrm{D}: \mathrm{s}=0.1$ & 7.67 & 5.88 & 1.20 & 0.52 \\
$\mathrm{D}: \mathrm{s}=-0.4$ & 6.9 & 5.93 & 1.96 & 0.76
\end{tabular}

\section{Choice of $k_{\perp}$ and the model for calculating the flux spectrum peak}

The correlation length of the turbulence is set both by the $k_{x}$ inherent in the $k_{\perp}$ formulation and also by $\left.k_{y}\right|_{\max }$. The magnetic shear dependence in equation 4 suggests (with $\alpha=0$ ) that the effective $k_{x}$ is lower at $s=0.1$ than at $s=1$ (for the same $\left.k_{y}\right|_{\max }$ ). This would correspond to increased correlation lengths as the magnetic shear is reduced. The $k_{x}$ and $k_{y}$ components of $k_{\perp}$ from equation 4 are plotted in figure 5 , for the GA-standard $s=1$ and $s=0.1$ case, where $\left\langle\theta^{2}\right\rangle$ is calculated from the QuaLiKiz eigenmode ansatz. At $s=1$ the assumed structures are approximately isotropic, particularly at lower, transport relevant $k_{y}$. At $s=0.1, k_{x}<k_{y}$. Even though $\left\langle\theta^{2}\right\rangle$ increases as the magnetic shear decreases, this does not compensate the decreasing $s^{2}$, at least down to $s=0.1$. This was corroborated by linear 
GENE analysis, where the eigenmodes were self-consistently calculated.

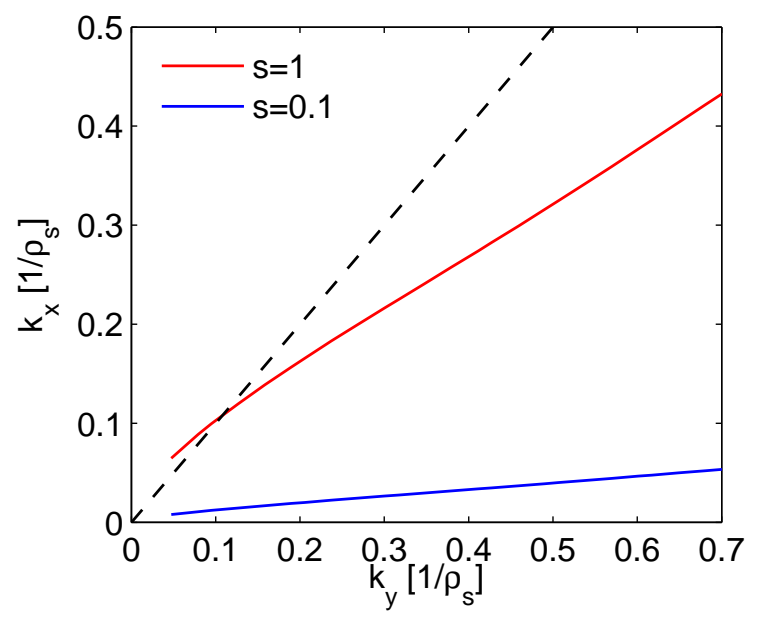

FIG. 5. $k_{x}$ as a function of $k_{y}$, as calculated in QuaLiKiz for $s=1$ and $s=0.1$, from the $k_{\perp}$ formulation given in equation 4 . The dashed curve represents $k_{x}=k_{y}$, as a measure for the degree of isotropy.

However, the magnetic shear dependence in equation 4 is not consistent with the nonlinear results, where the correlation length decreases with decreasing shear, as seen in table III. The eddy structure for both the $s=0.1$ and $s=1$ non-linear GA-standard cases are shown in figure 6 . The structures are roughly isotropic. The decreased correlation length at low magnetic shear is a result of the increased $\left.k_{y}\right|_{\max }$ at $s=0.1$.

Therefore the $\left.k_{y}\right|_{\max }$ calculation is also critical for setting correlation length and flux values. However, it was found that due to the large weight of $1 / k_{y}^{2}$ at low $k_{y},\left.k_{y}\right|_{\max }$ typically corresponds to the lowest (in $k_{y}$ ) unstable mode, in spite of the relatively low growth rates. For QuaLiKiz this is typically in the range of $k_{y}=0.05-0.1$. This is in disagreement with the non-linear flux spectra maxima from the magnetic shear scans, only agreeing approximately

FIG. 6. Contours of the electrostatic potential (top row) and the $\mathrm{x}$ and $\mathrm{y}$ length correlation functions (bottom row) for a typical timeslice in Gene $s=1$ (left column) and $s=0.1$ (right column) GA-standard case simulations. 
at $s=1$. Thus both the $k_{\perp}$ and $\left.k_{y}\right|_{\max }$ formulations must be rectified to correctly account for the magnetic shear dependencies on the flux in the mixing length model.

The non-linear flux spectra maxima, $\left.k_{y}\right|_{\max }$, were reproduced by replacing the effective $k_{x}$ in $k_{\perp}$ with the actual $k_{x}$ values from the non-linear simulations. Thus $k_{\perp}^{2}(n l)=k_{y}^{2}+$ $\left\langle k_{x}\right\rangle_{n l}^{2}\left(k_{y}\right)$, where $\left\langle k_{x}\right\rangle_{n l}\left(k_{y}\right)$ is the averaged value of $k_{x}$ for each $k_{y}$ mode in the non-linear simulation. $\left\langle k_{x}\right\rangle\left(k_{y}\right)$ is defined as $\sum k_{x}\left|\phi\left(k_{x}, k_{y}\right)\right|^{2} / \sum\left|\phi\left(k_{x}, k_{y}\right)\right|^{2}$, where the summation is over $k_{x} \geq 0$ for a given $k_{y}$. The values of $\left|\phi\left(k_{x}, k_{y}\right)\right|^{2}$ are pairwise averaged over negative and positive $k_{x}$, as well as over the parallel coordinate and for the times corresponding to the saturated state of the simulation. In the non-rotating, up-down symmetric plasmas considered in our simulations, $k_{x}$ is symmetric around 0 . In figure 7 these $\left\langle k_{x}\right\rangle$ values are plotted versus $k_{y}$ for the GENE non-linear GA-standard case magnetic shear scan. At low $k_{y}$, the eddies are not isotropic. $\left\langle k_{x}\right\rangle$ does not continue to drop at low $k_{y}$, and saturates at a value which increases as the magnetic shear decreases. This corresponds to reduced correlation lengths at low magnetic shear.



FIG. 7. $\left\langle k_{x}\right\rangle\left(k_{y}\right)$ for the $k_{y}$ points from a GENE non-linear GA-standard magnetic shear scan

The $\left.k_{y}\right|_{\text {max }}$ corresponding to the maximum $\gamma_{k} / k_{\perp}^{2}(n l)$ corresponds well with the non-linear flux spectra $k_{y}$ maximum, as seen in figure 8 for the entire range of non-linear simulations carried out. $\gamma_{k}$ was taken from GENE linear simulations and $k_{\perp}^{2}(n l)$ from GENE non-linear simulations. The considerations raised in sections III A - the stabilization of the toroidal branch at low $s k_{y}$ - also plays a role in setting $\left.k_{y}\right|_{\text {max }}$. Not only is $\left\langle k_{x}\right\rangle$ increased at low magnetic shear, but the growth rates themselves are reduced at low $s k_{y}$, further increasing 
the obtained $\left.k_{y}\right|_{\max }$, leading to reduced correlation lengths at low magnetic shear.
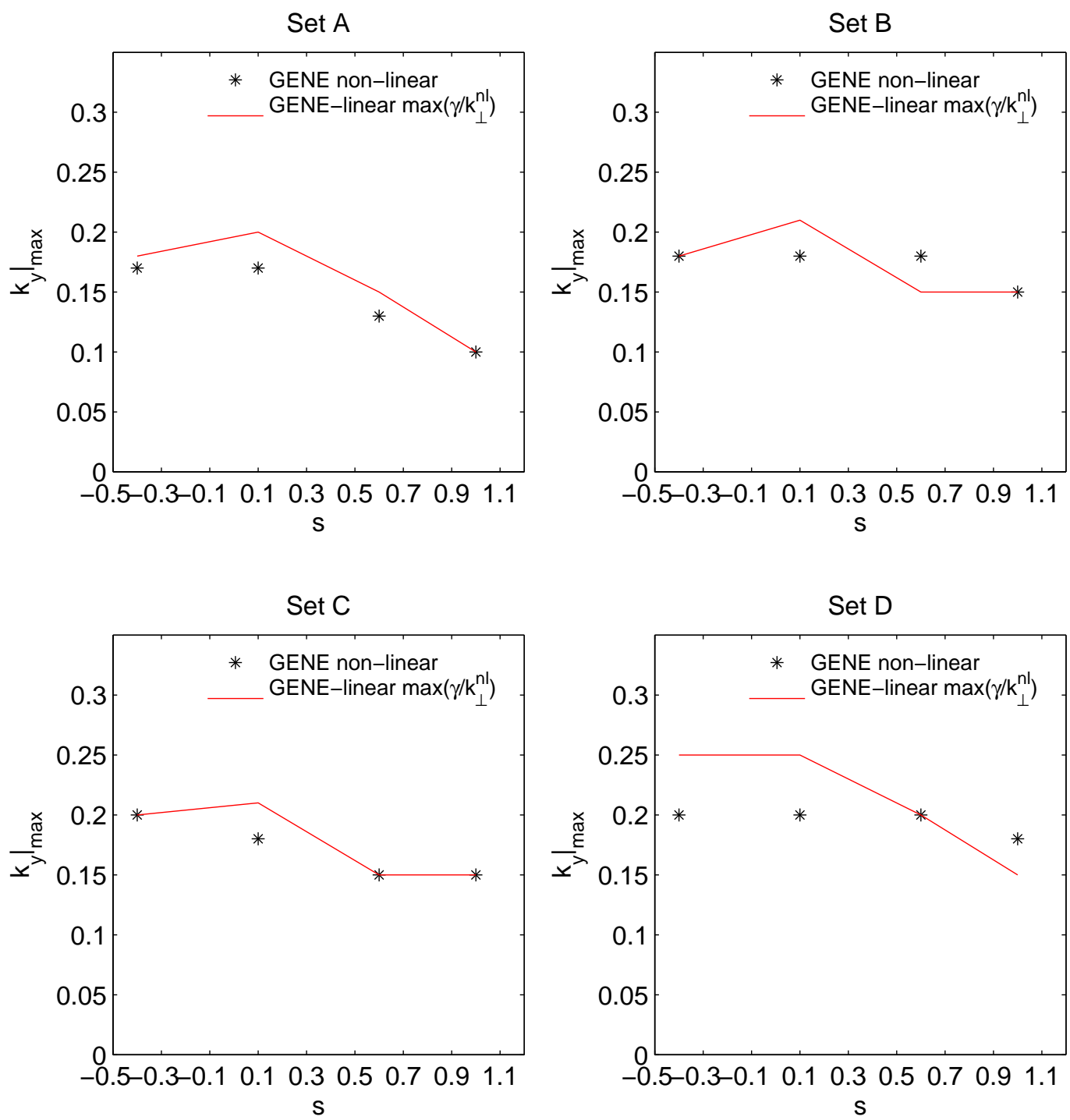

FIG. 8. Comparison of $\left.k_{y}\right|_{\text {max }}$ from non-linear simulations, and from the maximum of the $\gamma_{k} / k_{\perp}^{2}(n l)$ spectrum, where $\gamma_{k}$ is from GENE-linear calculations, and $k_{\perp}^{2}(n l)=k_{y}^{2}+\left\langle k_{x}\right\rangle^{2}\left(k_{y}\right)$ is taken from the non-linear simulations

In light of these observations from the non-linear simulations, we have improved the $k_{\perp}^{2}$ formulation in QuaLikiz by taking into account the non-linear contribution to $\left\langle k_{x}\right\rangle$. This simultaneously recovers the non-linear flux spectra $k_{y}$ maximum, and provides the magnetic shear and q-profile dependence on the precise relation between $k_{y}$ and $\left\langle k_{x}\right\rangle$ at $\left.k_{y}\right|_{\max }$. We have found that the following relation successfully reproduces the non-linear $\left\langle k_{x}\right\rangle\left(k_{y}\right)$ for the magnetic shear and q-profile scans carried out in this work: 


$$
\left\langle k_{x}\right\rangle\left(k_{y}\right)=k_{y} s \sqrt{\left\langle\theta^{2}\right\rangle}+0.4 \frac{e^{-2|s|}}{\sqrt{q}}+\frac{3}{2}\left(k_{y}-0.2\right) H\left(k_{y}-0.2\right)
$$

Where $H$ is a Heavyside function. The first term on the RHS is the effective $k_{x}$ due to the magnetic shear, which also appears in the previous $k_{\perp}^{2}$ formulation. The second term approximates the value of the non-linear contribution to $\left\langle k_{x}\right\rangle$ in the long wavelength, transport relevant region. The third term on the RHS was set to fit the non-linear $\left\langle k_{x}\right\rangle$ at higher $k_{y}$, and only plays a minor role at transport relevant $k_{y}$. The first term dominates at high magnetic shear, while the second term dominates at low magnetic shear. The QuaLiKiz mixing length rule formulation for $k_{\perp}^{2}$ was thus set in accordance with equation 5 . The results for $\left.k_{y}\right|_{\text {max }}$, using the QuaLiKiz $\gamma_{k}$ and the new $k_{\perp}^{2}=k_{y}^{2}+\left\langle k_{x}\right\rangle^{2}$, are seen in figure 9, where the results for the newly formulated $k_{\perp}^{2}$ are compared with the previously formulated $k_{\perp}^{2}$. The new formulation leads to a much improved reproduction of the non-linear $\left.k_{y}\right|_{\max }$. The absolute value $|s|$ in the exponent in the $\left\langle k_{x}\right\rangle$ formulation was set to avoid a non-physical exponential increase of $\left\langle k_{x}\right\rangle$ at large absolute values of negative magnetic shear. While the continuing decrease of the correlation length at negative magnetic shear is then not captured, we note that in general the QuaLiKiz predictions at negative magnetic shear deviate from the non-linear predictions due to an underprediction of the slab ITG branch. This is seen in figure 9 , for $s<\sim-0.3$, where the high values predicted for $\left.k_{y}\right|_{\max }$ is due to mode stabilization at lower $k_{y}$, and not due to $k_{\perp}^{2}$. The present formulation is thus relevant for low magnetic shear, while improved agreement between QuaLiKiz and non-linear flux predictions at negative magnetic shear is left for future work.

In addition to improving the $k_{\perp}$ formulation, we can consider also introducing a shearing effect of zonal flows on large wavelength turbulence ${ }^{28}$. This can be done by a ExB quench rule similar to that employed in the quasilinear models GLF23 ${ }^{29,30}$ and TGLF ${ }^{31}$ for external toroidal velocity. Indeed, the non-linear $\left.k_{y}\right|_{\max }$ was also recovered by calculating $\max \left(\gamma_{e f f} / k_{y}^{2}\right)$, where $\gamma_{e f f}$ is a quenched growth rate taken as $\gamma_{\text {eff }}=\max \left(0, \gamma_{k}-0.3 \omega_{E x B}\right)$. $\omega_{E x B}$ is the effective zonal flow ExB shear rate taken from the GENE non-linear simulations, including the effect of the autocorrelation time on the effective shearing time ${ }^{32}$. 0.3 is a tuned coefficient which provided a best fit to the non-linear flux spectra maxima throughout the set of simulations. $k_{\perp}$ was replaced by $k_{y}$ due to the approximate $k_{x}$ and $k_{y}$ isotropy. However, the simultaneous inclusion of $\gamma_{\text {eff }}$ and the improved $k_{\perp}$ formulation does not change the calculation of $\left.k_{y}\right|_{\max }$ compared to the sole inclusion of the improved $k_{\perp}$ formulation. This is 
Set A

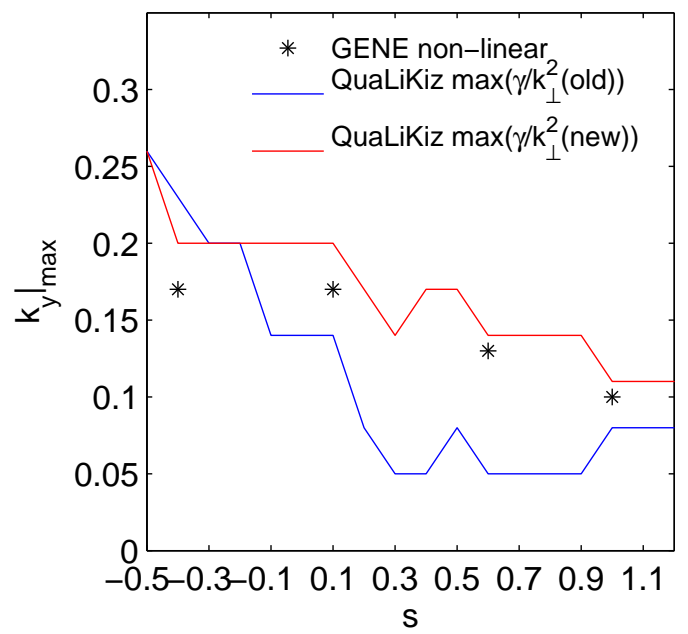

Set C

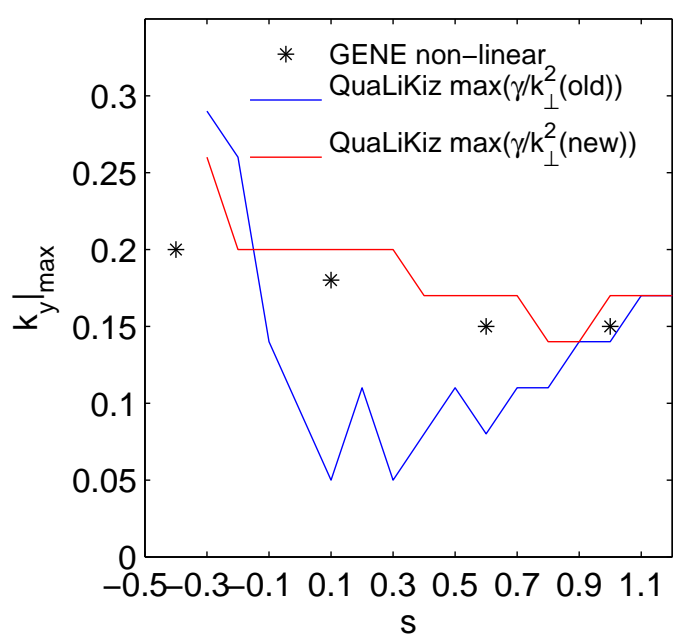

Set B

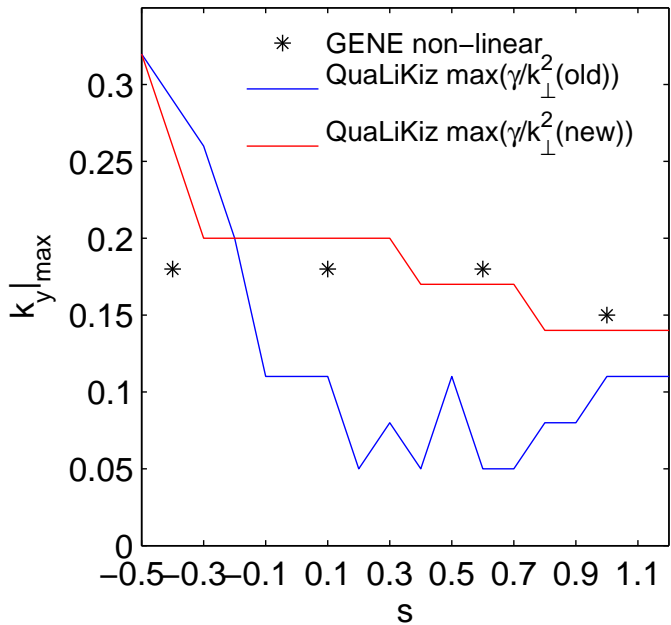

Set $D$

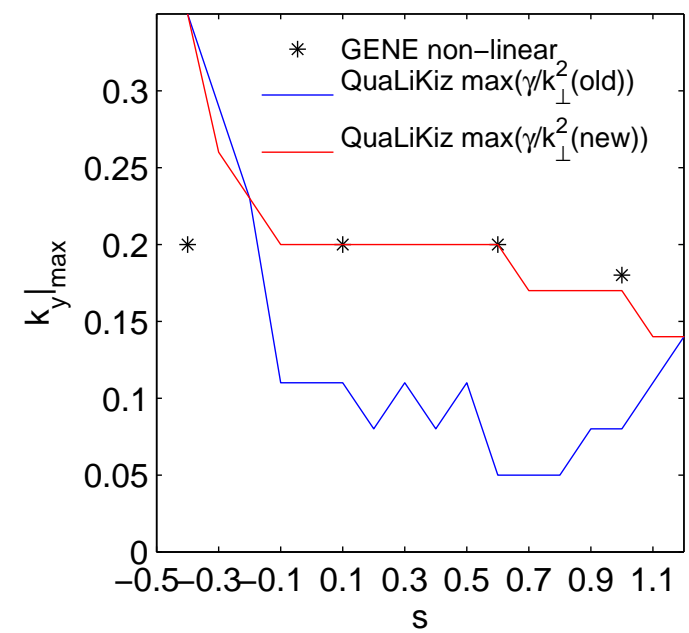

FIG. 9. Reproduction of the non-linear flux spectra maximum from the maximum of the $\gamma_{k} / k_{\perp}^{2}(n l)$ spectrum, where $\gamma_{k}$ is from QuaLiKiz, and $\left\langle k_{x}\right\rangle^{2}$ is taken from a formula found to reproduce Gene non-linear simulations

because the role played by the zonal flow shearing - the quenching of long wavelength modes - is effectively played by the non-isotropy of $k_{y}$ and $k_{x}$ at low $k_{y}$. Due to the challenge in parameterizing the zonal flow shear rate $\omega_{E x B}$ throughout parameter space within QuaLiKiz, and given the minor effect the zonal flow shearing of low $k_{y}$ modes has on determining $\left.k_{y}\right|_{\max }$ if the non-linear $\left\langle k_{x}\right\rangle$ is included in the model, it was decided not to include a zonal flow growth rate quench model in QuaLiKiz.

A possible mechanism for the dependence of magnetic shear on the zonal flow impact on 
$\left\langle k_{x}\right\rangle$ is now discussed. In non-linear mode coupling, the amount of energy that is injected into the primary mode is balanced by the coupling to other modes at the same scale or nearby scales. In general this coupling must satisfy a frequency matching condition as well as a wavenumber matching condition $\left(\omega_{p}+\omega_{q}-\omega_{k}=0, \mathbf{p}+\mathbf{q}-\mathbf{k}=0\right)$. At high magnetic shear, the modes are more strongly ballooned (more localized around $\theta=0$ ), and in addition the effective local radial wavenumber varies poloidally, since $k_{x}^{e f f} \sim k_{y} \hat{s} \theta+k_{x}$. This makes it rather difficult for the dominant ballooning eigenmode to couple effectively to a zonal mode, which is poloidally symmetric, with a direct interaction. However, in the case of low shear, the ballooning structure of the primary mode is much less pronounced, and in addition the local $k_{x}^{e f f}$ is a much weaker function of $\theta$. This makes it easier for the primary mode to couple directly to a zonal mode. This dependency of magnetic shear on zonal flows is well known and has been observed in a number of different contexts, for example in ETG gyrofluid simulations $^{33}$. In the direct simulations carried out in this work, the relative amplitude of zonal flows is indeed found to be greater at low magnetic shear, as seen in figure 10 for the set A (GA-standard case) $s=1$ and $s=0.1$ cases. The saturated electrostatic potential amplitudes are normalized, thus the lower normalized amplitude of the $s=0.1 k_{y}>0$ spectrum compared to the $s=1$ case reflects the higher relative amplitude of the $s=0.1$ $k_{y}=0$ modes (zonal flows) compared with the $s=1$ case.

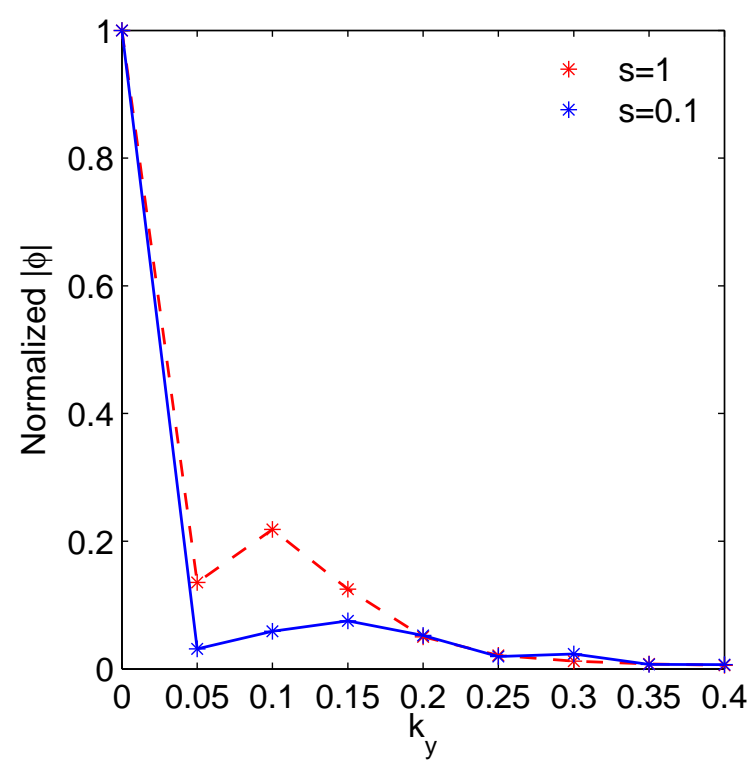

FIG. 10. Relative amplitude of zonal flow modes $\left(k_{y}=0\right)$ compared with the $k_{y}>0$ modes for the GA-standard $s=1$ and $s=0.1$ cases. $k_{x}$ has been averaged over at each $k_{y}$. 
Since in the case of wave interactions it takes at least three waves to interact, when the direct interaction with the zonal flow is allowed, another drift mode with non-zero $k_{x}$ also extracts energy from this interaction (in fact enstrophy and not energy is exchanged, but for the sake of current argument this is not of essential importance). This has two effects on turbulence. First, the zonal flows isotropize turbulence, since they can shear streamers apart. They also reduce the degree of ballooning in the mode structure. This isotropization can make the non-linearly formed $k_{x}$ important in the computation of the flux or the mixing length saturation level. At high magnetic shear, the 'non-linear' $k_{x}$ is less important, since the zonal flow drive is lessened due to effective $k_{x}$ being a function of $\theta$. But at low magnetic shear, the turbulence is isotropized due to zonal flows, so a substantial amount of the energy is found in $k_{x} \neq 0$ fluctuations.

In summary, the previous $k_{\perp}^{2}$ formulation in QuaLiKiz led both to an incorrect estimate of the non-linear flux spectrum maximum, and to the wrong dependency on magnetic shear in the $k_{\perp}^{2}$ description itself at low magnetic shear. From analysis with non-linear and linear GENE simulations, it was found that improving the $k_{\perp}^{2}$ formulation in the QuaLiKiz mixing length rule, taking into account the $\left\langle k_{x}\right\rangle\left(k_{y}\right)$ found from non-linear simulations, leads to an improved estimate of $\left.k_{y}\right|_{\max }$ and the magnetic shear and q-profile dependence of $k_{\perp}^{2}$. The increased values of $\left\langle k_{x}\right\rangle\left(k_{y}\right)$ at low magnetic shear at low, transport relevant, $k_{y}$ values may be related to the observed greater relative amplitude of zonal flow modes. This may rise due to the less pronounced ballooning structure of the low magnetic shear eigenmodes. It is also possible that linearly unstable modes with $k_{x}$-center $\neq 0$ - which are more prevalent at low magnetic shear - further contribute to the $k_{x}$ spectrum.

\section{Frequency broadening}

In the QuaLiKiz model the width of the broadened electrostatic field frequency function due to stochastic scattering is taken as the linear growth-rate, $\gamma_{k}$. This assumption can be checked by comparison to the non-linear frequency spectra from GENE. The width of the non-linear frequency spectra, calculated for each $k_{y}$, is compared to the linear growth rate from linear GENE. In figure 11 we see the examples of the frequency broadening data and Lorentzian fit from set A (GA-standard case) $s=1$ and $s=0.1$ cases for $k_{y}=0.2$. The GENE calculated linear frequency $\left(\omega_{l i n}\right)$ and growth rate $\left(\gamma_{l i n}\right)$ are compared with the 
non-linear frequency spectrum center $\left(\omega_{f i t}\right)$ and width $\left(\Delta \omega_{f i t}\right)$ as defined by the Lorentzian fit. We note that in analysis of GYRO frequency spectra, both Lorentzian and Gaussian fits to the frequency spectra were satisfactory in recovering the FWHM, while a T-model was in fact the best fit to the data ${ }^{10}$. In figure 12 the comparison between the non-linear frequency spectra width (from the Lorentzian fit) and the linear growth rate is shown for all the sets at $s=1, s=0.6$, and $s=0.1$. The assumption generally holds for $s=1$ and $s=0.6$ in the transport-relevant range of $k_{y}<0.3$. However, it is very interesting to note that for $s=0.1$, the frequency width does not equal the linear growth rate for any value of $k_{y}$. In the transport relevant range $0.15<k_{y}<0.5$, the non-linear frequency width is significantly higher than the linear growth rate estimation. This is consistent with the generally lower autocorrelation times observed at low magnetic shear, as seen in table III. This must be taken into consideration in the quasi-linear model.
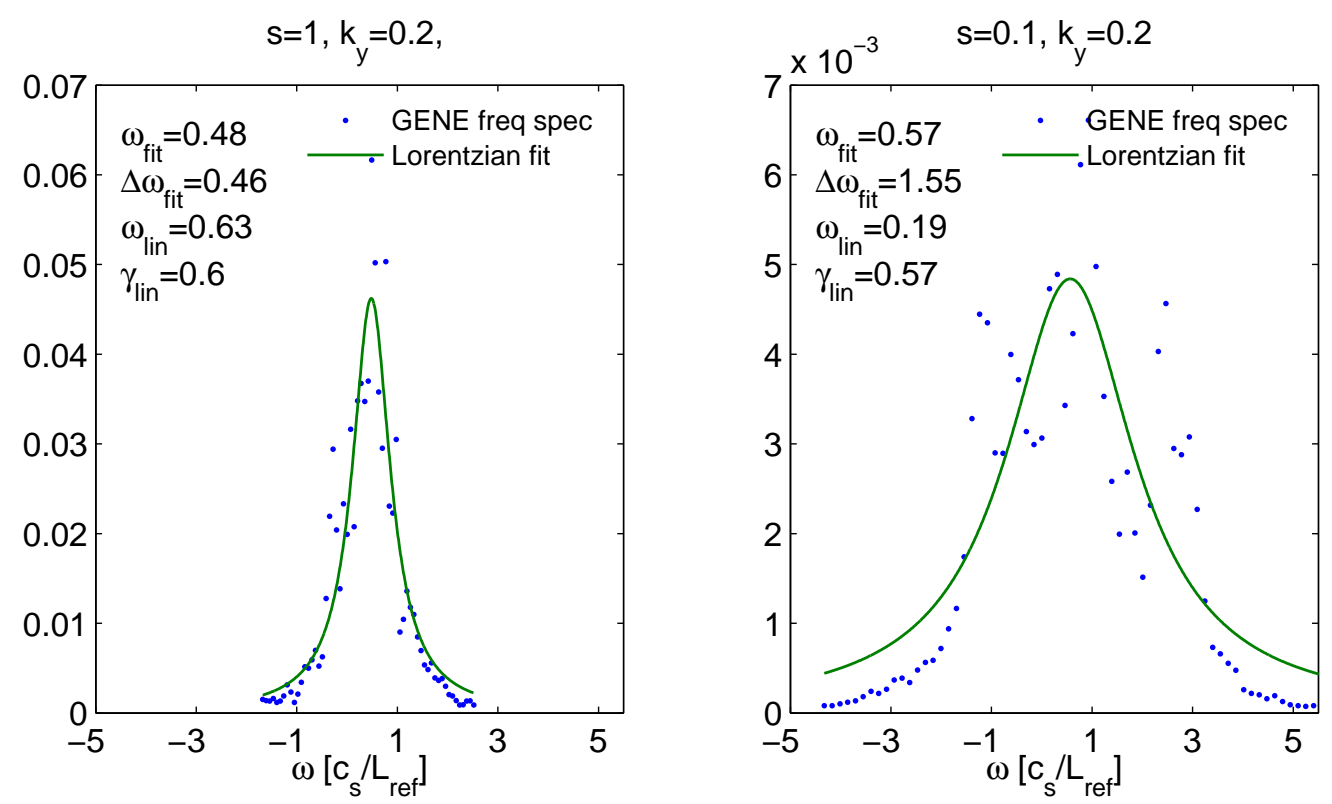

FIG. 11. Frequency spectra for $k_{y}=0.2$, averaged over saturated periods of GENE non-linear simulations from set A with $s=1$ (left panel) and $s=0.1$ (right panel)

This increased frequency broadening at low magnetic shear may be a result of non-linear decorrelation mechanisms, such as zonal flows, which play a stronger role at low magnetic shear as discussed in section IV C 1. Also in the set with lowest flux, set D, where the impact of zonal flows is also expected to be stronger due to the vicinity to the instability threshold, the frequency broadening is greater than the linear growth rate estimation even at $s=0.6$ 

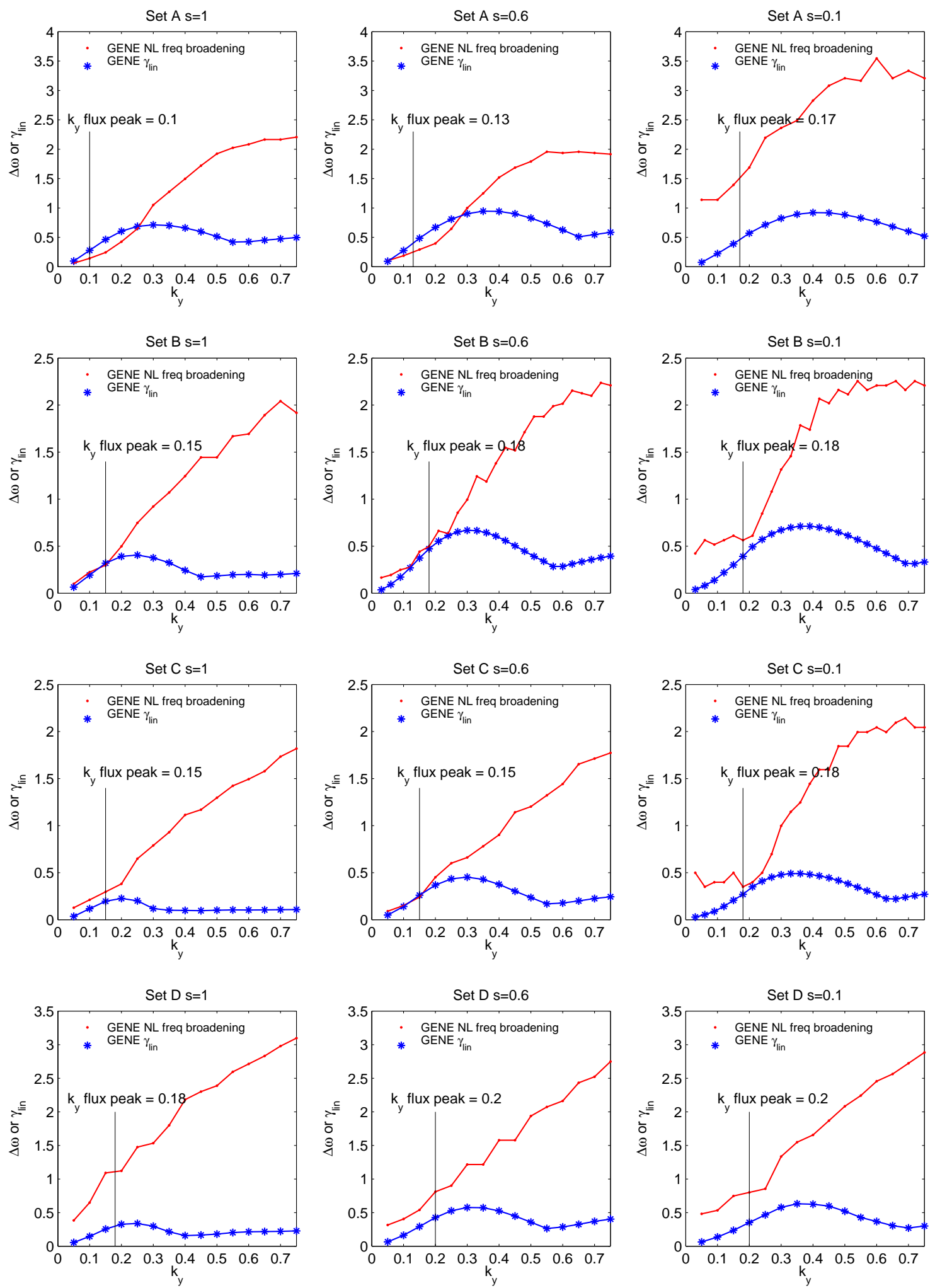

FIG. 12. Non-linear frequency spectra fitted Lorentzian width and linear growth rates are compared for a range of $k_{y}$, for all 4 sets studied, for $s=1$ (left column), $s=0.6$ (middle column), and $s=0.1$ (right column) 
and $s=1$. The existence of zonal flows affects the response function. This can be seen via a renormalization of the wave-kinetic equation. In the presence of zonal flows, the basic wave-kinetic response function takes the form:

$$
R=\frac{R_{0}}{\left[1+R_{0}^{2}\left(\frac{\partial}{\partial r}\left[V_{E}(r) k_{y}\right]\right)^{2} \frac{1}{N} \frac{\partial^{2} N}{\partial k_{x}^{2}}\right]}
$$

where $N$ is the wave action. Using this form instead of the linear response function, we obtain:

$$
\left|\Phi_{k, \omega}\right|^{2} \propto \frac{\left|\Phi_{k}\right|^{2}}{\left(\omega-\omega_{k}\right)^{2}+\gamma_{k}^{2}+\alpha\left(\frac{\partial}{\partial r} V_{E}(r)\right)^{2}}
$$

where $V_{E}(r)$ is the zonal flow velocity, and $\alpha \equiv \frac{k_{y}^{2}}{\left|\Phi_{k}\right|^{2}} \frac{\partial^{2}\left|\Phi_{k}\right|^{2}}{\partial k_{x}^{2}}$. This equates to increased frequency broadening, beyond the linear growth rate assumption. Such a reduction of the fluxes due to perpendicular flow shear was also employed in a transport model by Hinton and Staebler ${ }^{34}$.

We define the ratio between the frequency broadening from the non-linear simulations and the linear growth rate, at each magnetic shear value, as the weighted average $\left\langle\Delta \omega_{k} / \gamma_{k}\right\rangle$, with the weight set by the $\phi\left(k_{y}\right)$ amplitude spectrum. For the entire collection of simulations, $\left\langle\Delta \omega_{k} / \gamma_{k}\right\rangle_{s=0.1} /\left(\frac{1}{2}\left\langle\Delta \omega_{k} / \gamma_{k}\right\rangle_{s=1}+\frac{1}{2}\left\langle\Delta \omega_{k} / \gamma_{k}\right\rangle_{s=0.6}\right)=2.5$, averaging over all four sets. This reflects the increased frequency broadening at low magnetic shear, which is highest for set A (GA-standard case) and lowest for set D. In the absence of more rigorous theory, we have simply included an additional shear dependent normalization factor to the fluxes calculated by QuaLiKiz, in the form of $2.5(1-|s|)$ for $|s|<0.6$. This normalization factor roughly captures the effect of the decreased autocorrelation time due to increased frequency broadening (possibly due to zonal flows) at low magnetic shear.

The increase in the frequency broadening is also correlated with a reduction in the effective non-linear transport weight. This is seen by comparing the ratio of the quasilinear transport weight (flux over spectral intensity as a function of $\mathrm{k}$ ) with the non-linear transport weight, defined as an overage ${ }^{35}$. These overages were found to be $\sim 1.4$ - at transport relevant $\mathrm{k}$ values - for a variety of different cases. When calculating this overage for the $\mathrm{s}=0.1 \mathrm{GA}-$ standard case, as seen in figure 13, we however obtain an increased overage compared to the $\sim 1.4$ value found for the $\mathrm{s}=1$ case. This overage increase, corresponding to the deviation of the quasilinear flux from the non-linear flux due to the non-constancy of the transport weight, corresponds well with our ad-hoc correction factor from the frequency broadening. 
An additional possible explanation for the non-unity overage could be the non-linear transfer of energy from unstable eigenmodes to stable eigenmodes. This is proposed to lead to a similar factor $\sim 1.4$ between quasilinear and non-linear fluxes ${ }^{36}$. This is because the construction of the quasilinear flux assumes that the unstable mode contributes fully to the electrostatic potential amplitude. However, in the non-linear case, linearly damped modes take up a given proportion of the total electrostatic potential amplitude. The net contribution of these linearly damped modes to the flux is typically much reduced compared with the dominant unstable mode. Thus, since the transport weight (following a $k_{y}$ decomposition) is defined as the flux divided by the electrostatic potential, the non-linear weight is expected to be lower than the quasilinear weight due to the existence of the low-flux-contributing linearly damped modes which do contribute to the saturated electrostatic potential amplitude. An increased proportion of linearly damped modes in the low magnetic shear system would then be consistent with increased transport weight overage. A broadened frequency spectrum could also be a signature of an increased spectrum of modes in the system. The increase of zonal modes - as observed at low magnetic shear - is expected to lead to increased coupling to $n>0$ linearly damped modes. Indeed, as seen in figure 14, singular value decompositions of the saturated electrostatic potential of the GA-standard $s=1$ and $\mathrm{s}=0.1$ cases show that the $\mathrm{s}=0.1$ case has a much broader spectrum of linearly stable modes beyond the dominant unstable mode. This method of analysis is outlined in Hatch et $\mathrm{al}^{37}$. The existence of a broader spectrum of linearly damped modes is consistent with a higher transport weight overage. However, we also note that at the current level of analysis it is not possible to separate in the singular value decomposition linearly damped modes from linearly unstable modes with midplane $k_{x 0} \neq 0$ (related to the conventional ballooning angle through $\left.k_{x 0}=k_{y} \theta_{0} s\right)$. While the $k_{x 0}=0$ modes are dominant for both $\mathrm{s}=0.1$ and $\mathrm{s}=1$, the $k_{x 0}$ growth rate spectrum is broader for $\mathrm{s}=0.1$, when compared with $\mathrm{s}=1$. These modes could in principle also contribute to the frequency broadening. More analysis is necessary in this respect.

In summary, at low magnetic shear the non-linear frequency broadening is observed to be larger than the linear growth rate width assumption. This broadening is correlated with an increase in the quasilinear to non-linear transport weight overage. An ad-hoc magnetic shear dependent normalization factor, based on the degree of increased non-linear frequency broadening at low magnetic shear, has been introduced into QuaLiKiz. The physical expla- 
nation of the frequency broadening and associated transport weight overage increase may be related to the relative increase in the zonal flow amplitude at low magnetic shear. A direct renormalization of the linear response function due to zonal flows is suggested. An additional explanation may be the increased non-linear coupling (via zonal flows) to linearly damped modes. More theoretical work is necessary to more fully uncover the mechanism of the increased frequency broadening and transport weight overage increase at low magnetic shear. However, on a more pragmatic level, the QuaLiKiz and non-linear flux prediction comparison is much improved through the combination of the $k_{\perp}$ formulation improvement and the ad-hoc magnetic shear dependent normalization. This is shown in the following section.

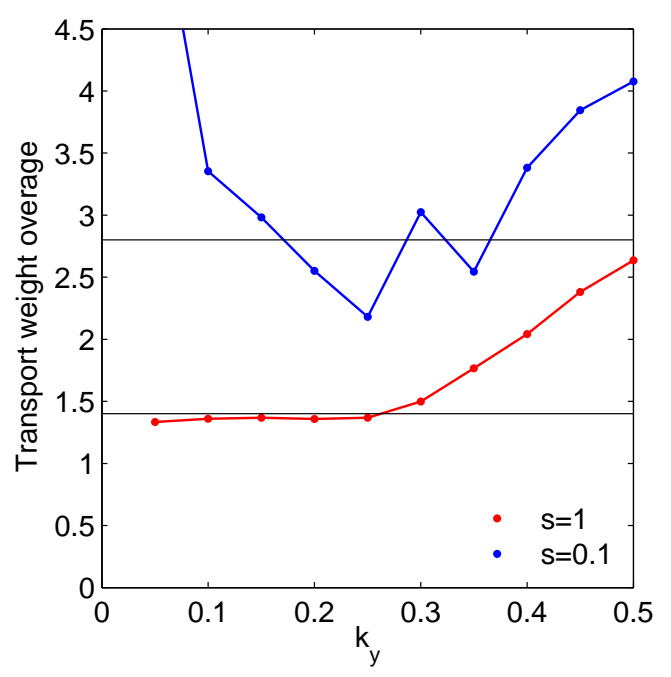

FIG. 13. Ratio of quasilinear to non-linear transport weights for the GA-standard $s=1$ and $s=0.1$ cases

\section{Comparison of QuaLiKiz and non-linear flux predictions}

Having taken into account both modifications discussed above, the $k_{\perp}^{2}$ alteration and frequency broadening renormalization, we now compare the particle and heat flux predictions from the modified QuaLiKiz and the non-linear simulations, for all GENE runs carried out, as well as GyRo for the GA-standard magnetic shear, q-profile, and $R / L_{T}$ scans. The results are displayed in figure 15. The agreement with the GA-standard case s-scan is much improved compared to the previous mixing length formulation shown in figure 1. Simul- 


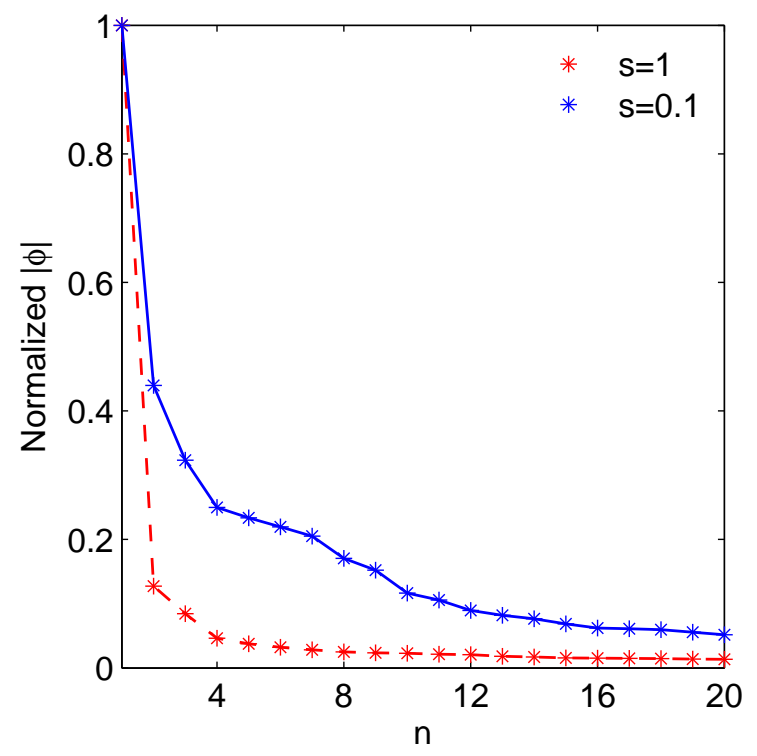

FIG. 14. Singular value spectrum of mode amplitudes calculated from the GA-standard s=1 and $\mathrm{s}=0.1$ saturated electrostatic potentials

TABLE IV. RMS errors between QuaLiKiz and non-linear simulation predicted fluxes, for all GENE and GYRO runs shown in figure 15

\begin{tabular}{c|c|c|c} 
& $\sigma_{\chi_{i}}$ & $\sigma_{\chi_{e}}$ & $\sigma_{D}$ \\
\hline QuaLiKiz09 & 1.13 & 1.53 & 1.84 \\
\hline With new $k_{\perp}^{2}$ only & 0.52 & 0.77 & 1.24 \\
\hline With new $k_{\perp}^{2}$ and frequency broadening renormalization & 0.26 & 0.33 & 1
\end{tabular}

taneously, the agreement with the q-scan and $R / L_{T}$ scan is maintained. When exploring additional areas in parameter space, i.e. the B, C, and D sets, reasonable agreement is also observed. These results are summarized in table IV, where the RMS error between the particle and heat fluxes between QuaLiKiz and the non-linear Gene and Gyro simulations are listed. The RMS error is defined as $\sigma=\sqrt{\sum_{i} \epsilon_{i}^{2} / \sum_{j} \chi_{j(N L)}^{2}}$, where $\epsilon_{i}$ is the diffusivity or flux difference between QuaLiKiz and the non-linear prediction for a given simulation, and $\chi_{j(N L)}$ the diffusivity or flux from the non-linear simulation. The RMS errors obtained using the previous QuaLiKiz version, correcting only $k_{\perp}^{2}$, and both correcting $k_{\perp}^{2}$ and including the frequency broadening renormalization are all compared. Both modifications are of comparable importance. 

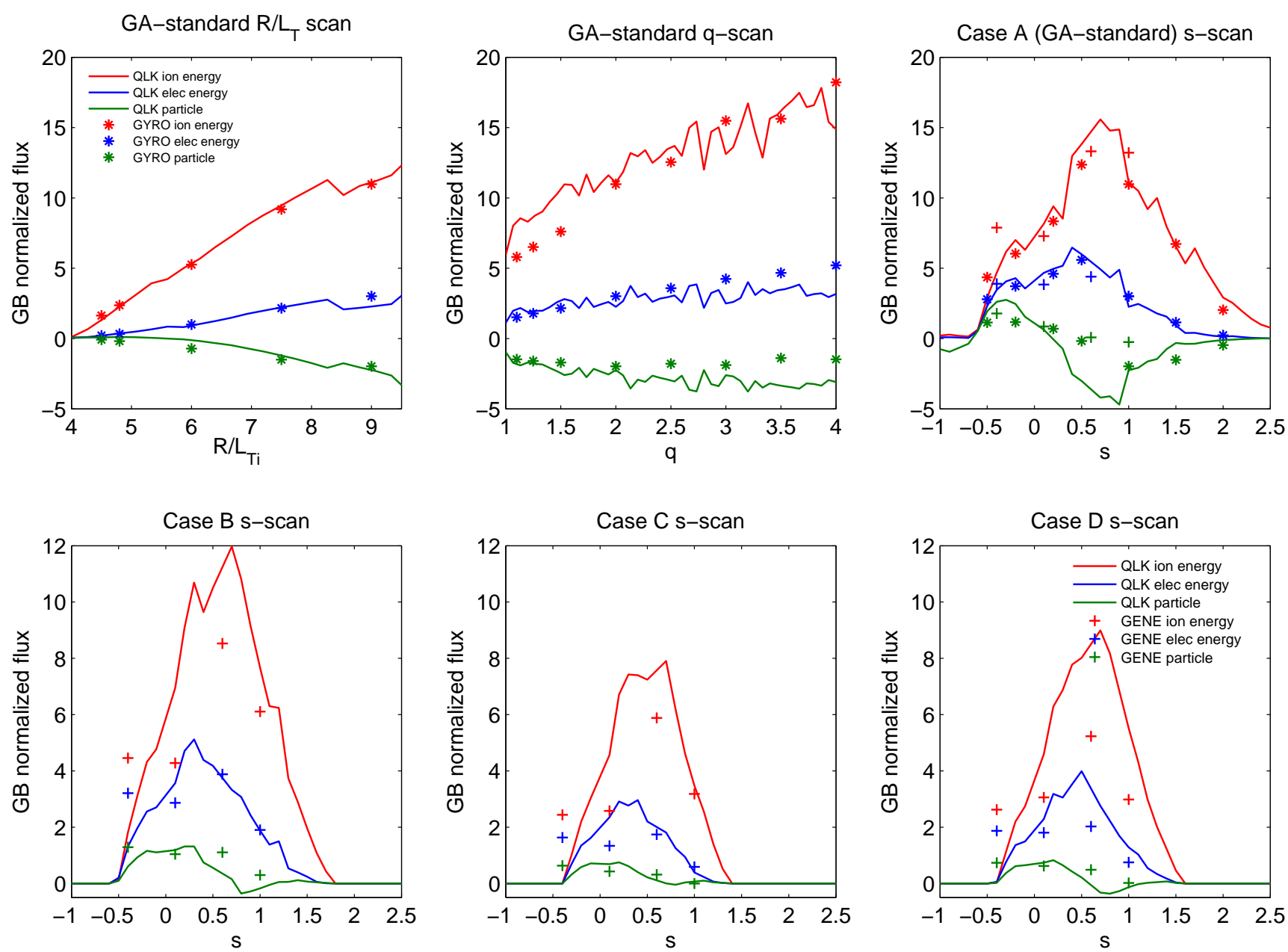

FIG. 15. Comparison of the altered QuaLiKiz model with the 4 sets of Gene runs, and with the Gyro GA-standard case s-scan, q-scan, and $R / L_{T}$ scan.

\section{DISCUSSION AND CONCLUSIONS}

Quasilinear flux predictions in the previous version of QuaLiKiz did not agree with GYRO non-linear flux predictions at low magnetic shear. This work has systemically examined the assumptions underlying the QuaLiKiz model, to uncover the physics behind this discrepancy. Improvements in the model where implemented, guided and validated by a further set of non-linear simulations carried out with GENE. This led to a significant improvement in the comparison between QuaLiKiz and non-linear simulations.

It was found that the previous formulation of $k_{\perp}^{2}$ in the QuaLiKiz mixing length rule for the saturated potential did not reproduce the magnetic shear dependence observed in nonlinear simulations, neither in the $\left.k_{y}\right|_{\max }$ of the flux spectrum, nor in the relation between $\left\langle k_{x}\right\rangle$ 
and $k_{y}$. Furthermore, the frequency broadening at low magnetic shear was observed in the non-linear simulations to be greater than the linear growth rate broadening assumption. This is correlated with a increase in the ratio between the quasilinear and non-linear transport weights (transport weight overage), which must be taken into account in the model.

The QuaLiKiz mixing length rule was thus improved by introducing a formula for $\left\langle k_{x}\right\rangle\left(k_{y}\right)$ in the $k_{\perp}$ formulation, which captures the magnetic shear and q-profile dependence from the non-linear simulations. This successfully reproduces the non-linear $\left.k_{y}\right|_{\text {max }}$ from the maximum of the $\gamma_{k} / k_{\perp}^{2}$ mixing length spectrum, and also captures the degree of isotropy between $\left\langle k_{x}\right\rangle$ and $k_{y}$, setting the precise value of the correlation length. Furthermore, a renormalization based on the weighted average of the increased frequency broadening was implemented, which was consistent with the observed degree of increase in the transport weight overage. These modifications led to a much improved agreement between the new version of QuaLiKiz, and non-linear GYRO and GENE magnetic shear scans for various values of $R / L_{T}, q$, and $T_{i} / T_{e}$.

We note that the reproduction of $\left.k_{y}\right|_{\max }$ can also be achieved by invoking zonal flow quenching of large wavelength turbulence in the mixing length rule. This results in reduced correlation lengths at low magnetic shear due to the stabilization of the toroidal ITG branch at combined low s and $k_{y}$, which leads to reduced growth rates at low $s k_{y}$. This stabilization was discussed when summarizing linear analytical and numerical results at low magnetic shear. In spite of the lower ITG threshold at low magnetic shear, the $\gamma$-stiffness is also lower at low magnetic shear. At low $k_{y}$ this is particularly the case, due to toroidal ITG branch stabilization at low $s k_{y}$. Since the $\left\langle k_{x}\right\rangle$ dependence on $k_{y}$ also depends on zonal flows, these two approaches for altering the mixing length rule stem from the same physics.

As detailed in Appendix B and Appendix C, the intrinsic assumptions in the QuaLiKiz model were also examined. It was found that locality holds down to $s=0.1$. The lowest order ballooning representation is also valid down to $s=0.1$, following comparisons of the QuaLiKiz fluid eigenmode assumption and GENE linear eigenfunction solutions. The quasilinear approximation also holds at low magnetic shear. This was verified by calculating the Kubo numbers from the entire set of non-linear GENE simulations. It was found the $K<1$ also at low magnetic shear. This strengthens the claim that the quasilinear/non-linear discrepancy at low magnetic shear is purely due to the formulation of the saturated potential intensity and frequency spectrum - which is outside of the scope of the quasilinear model itself - while 
the fundamental formulation of quasilinear diffusivities is still valid.

Future work on QuaLiKiz will be directed on reproducing the effect of external ExB shear and introducing a parameterization for shape effects beyond the $s-\alpha$ model. Furthermore, from the magnetic shear scans, we can see that ITG-TEM modes are fully stabilized at

high magnetic shear. These correspond to experimentally relevant values towards the edges of tokamak plasmas. This is not consistent with density fluctuation measurements which increase strongly with $\mathrm{r} / \mathrm{a}$. Present quasilinear models based purely on ITG-TEM-ETG modes tend thus to underpredict the level of transport towards the edge, compared to experimental measurements, if not constrained to a typical range of $r / a<\sim 0.8$. Resolving this issue is critical for fully predictive modeling of L-modes up to the separatrix, or for the region close to an H-mode pedestal.

Finally, the improved agreement in predicted fluxes between the present version of QuaLiKiz and the non-linear simulation magnetic shear scans were not only seen in the high flux GA-standard case, but also in lower flux cases closer to experimental parameters. This is encouraging for the future experimental validation of the QuaLiKiz model and for extrapolation to ITER. Transport in ITER at low magnetic shear will be important for all scenarios, due to the predicted ITER sawtooth period being significantly higher than the energy confinement time.

\section{ACKNOWLEDGMENTS}

This work, supported by the European Communities under the contract of Association between EURATOM/FOM and EURATOM/CEA, was carried out within the framework of the European Fusion Programme with financial support from NWO. The views and opinions expressed herein do not necessarily reflect those of the European Commission. This work is supported by NWO-RFBR Centre-of-Excellence on Fusion Physics and Technology (Grant nr. 047.018.002). The authors would like to thank: T. Görler, F. Merz, D. Told and H. Doerk for their support in using Gene; R. Waltz, J. Candy, P. Mantica, G. Staebler and C. McDevitt for fruitful discussions; J. Kinsey for having providing access to GYRO simulation results. This research used resources of the National Research Scientific Computing Center, which is supported by the Office of Science of the U.S. Department of Energy under Contract No. DE-AC02-05CH11231. The authors are extremely grateful to D. Mikkelsen for having 
provided computational resources.

\section{Appendix A: Analytic fluid limit}

The analytic fluid limit is obtained by considering mode frequencies far above the resonance, i.e. $\omega \gg \omega_{d}, k_{\|} V_{\| i}$. This corresponds to driving gradients far above the linear thresholds. We assume an ordering $k_{\perp} \rho_{e} \ll k_{\perp} \rho_{i} \ll 1$, and $k_{\perp} \delta_{e} \ll k_{\perp} \delta_{i} \ll 1$, as well as adiabatic passing electrons, large-aspect-ratio, $\alpha=0$, and strongly ballooned eigenfunctions centered around the equatorial midplane. The fundamental linear gyrokinetic dispersion equation can then be simplified to the following ${ }^{14}$ :

$$
\left[1+\frac{n^{2} \omega_{p e}^{*} \omega_{d}\left(f_{t}+\frac{\tau}{Z}\right)}{f_{p} \omega^{2}}-\frac{n \omega_{n e}^{*}}{\omega}+\frac{n \omega_{d}}{\omega}+\left(-\frac{k_{\|}^{2} c_{e f f}^{2}}{2 \omega^{2}}+\frac{k_{\perp}^{2} d_{e f f}^{2}}{2}\right)\left(1+\frac{\tau}{Z} \frac{n \omega_{p e}^{*}}{\omega}\right)\right] \tilde{\phi}=0
$$

Where $\rho_{i, e}$ and $\delta_{i, e}$ are the particle Larmor radii and banana widths, $n$ is the toroidal wave number, $\omega_{d}=-\frac{k_{\theta} T_{s}}{e_{s} B}(\cos \theta+s \theta \sin \theta)$ is the vertical drift frequency, $\omega_{n e}^{*}=-\frac{k_{\theta} T_{s}}{e_{s} B} \frac{1}{L_{n}}$ and $\omega_{p e}^{*}=-\frac{k_{\theta} T_{s}}{e_{s} B} \frac{1}{L_{p}}$ are the diamagnetic frequencies associated with the density and pressure gradient lengths respectively, $V_{\| i}$ is the ion parallel velocity, $\tau=\frac{T_{i}}{T_{e}}, Z$ is the effective ion charge, $f_{t, p}$ are the trapped and passing particle fractions respectively, $c_{e f f}^{2} \equiv \frac{T_{e}}{m_{p}}$, and $d_{e f f}^{2} \equiv \frac{f_{p}}{f_{c}} \frac{T_{e}}{n_{e}} \sum_{i} \frac{n_{i} Z_{i}^{2}}{T_{i}} \delta_{i}+\frac{4 T_{e} m_{p}}{e^{2} B^{2}}$. In the limit where $q(r) \approx q\left(r_{0}\right)+q^{\prime}\left(r_{0}\right) x$, we can write $k_{\|}=$ $k_{\|}^{\prime} x=k_{\theta} x / L_{s}$, where $x \equiv\left(r-r_{0}\right)$ (the distance from the rational surface) and $L_{s} \equiv R q / s$. Transforming the radial coordinate from Fourier space to real space, we can also write $k_{\perp}^{2}=k_{\theta}^{2}-d^{2} / d x^{2}$. Equation $\mathrm{A} 1$ is then a second order differential equation for $\tilde{\phi}(x)$. A

Gaussian solution, $\tilde{\phi}=\phi_{0} e^{-\frac{x^{2}}{2 w^{2}}}$, with $w^{2}=-\frac{i \omega d_{e f f} L_{s}}{k_{\theta} c_{e f f}}$, implies that $\tilde{\phi}^{\prime \prime}=-\left(\frac{1}{w^{2}}+\frac{1}{w^{4}} x^{2}\right) \tilde{\phi}$, and thus imposes a quadratic equation for the solution of the growth rate $\gamma=n \operatorname{Im}(\Omega)$, with $\Omega=\omega / n$ :

$\Omega^{2}\left(1+\frac{k_{\theta}^{2} d_{e f f}^{2}}{2}\right)+\Omega\left(-\omega_{n e}^{*}+\omega_{d}+i \frac{k_{\theta}}{n L_{s}} \frac{c_{e f f} d_{e f f}}{2}+\frac{k_{\theta}^{2} d_{e f f}^{2}}{2} \frac{\tau}{Z} \omega_{p e}^{*}\right)+\frac{\omega_{p e}^{*} \omega_{d}}{f_{p}}\left(f_{t}+\frac{\tau}{Z}\right)+\frac{\tau}{Z} \omega_{p e}^{*} i \frac{k_{\theta} c_{e f f} d_{e f f}}{2 n L_{s}}=0$

To recover the interchange limit, where curvature dominates, we take the ordering $f_{p}, f_{t}, \frac{\tau}{Z}, \sim O(1)$, $k_{\theta} d_{e f f}, L_{p} / R, \frac{\left(q R / \epsilon^{0.5}\right)}{L_{s}} \sim O(\delta), L_{p} / L_{n} \sim O\left(\delta^{2}\right)$, where $\epsilon$ is the inverse-aspect-ratio. In this limit, we obtain for the growth rate:

$$
\gamma^{2}=\frac{\left(f_{t}+\frac{\tau}{Z}\right) n^{2} \omega_{p e}^{*} \omega_{d}}{f_{p}}
$$


While for the slab limit, the relevant ordering is $f_{p}, f_{t}, \frac{\tau}{Z}, \sim O(1), k_{\theta} d_{e f f}, L_{p} / R, \frac{L_{s}}{\left(q R / \epsilon^{0.5}\right)} \sim O(\delta)$, $L_{p} / L_{n} \sim O\left(\delta^{2}\right)$, which leads to the following expression for the growth rate:

$$
\gamma^{2}=\frac{\tau}{Z} \frac{n \omega_{p e}^{*} k_{\theta} d_{e f f} c_{e f f}}{2 L_{s}}
$$

Let us examine the magnetic shear dependence in each of the above limits. For the interchange limit, the s-dependence can be found in $\omega_{d}$, where $\omega_{d} \propto \cos \theta+s \theta \sin \theta \Rightarrow \gamma^{2} \propto$ $\cos \theta+s \theta \sin \theta$. In the slab limit, since $L_{s} \equiv R q /|s|$, we have $\gamma^{2} \propto|s|$. In both cases, the growth rates increase with magnetic shear. The fact that the growth rates increase with $s$ in the limit far above the linear instability threshold, while simultaneously the ITG instability threshold also increases with $s$, points towards reduced growth rate stiffness at lower magnetic shear.

\section{Appendix B: Locality approximation at low magnetic shear}

The QuaLiKiz model is a local code (as are the versions of non-linear Gyro and GEnE used here in this work), and thus assumes constant profile gradient lengths. This can be formulated as $\max (w, d)<\min \left(L_{n(e, i)}, L_{T(e, i)}\right)$, where $w$ is the mode width, $d$ the distance between rational surfaces, and $L_{n}, L_{T}$ the electron or ion density and temperature gradient lengths respectively. This assumption must fail at a sufficiently low value of $s$ as the distance between rational surfaces grows further and further apart, since $d=1 / s k_{\theta}$. At low shear $d$ and $w$ are comparable in the QuaLiKiz model (see Appendix A in ${ }^{9}$ ). We can estimate this $s$ value as follows: firstly since $d$ and $w$ are comparable at low shear, we find the $s$ for which $d=L_{T}$. $\frac{R}{d}=\frac{R}{L_{T}} \Rightarrow s k_{\theta} R=\frac{R}{L_{T}} \Rightarrow s\left(k_{\theta} \rho_{s}\right) \frac{R}{\rho_{s}}=\frac{R}{L_{T}}$. Taking $k_{\theta} \rho_{s}=0.1$, which is approximately the value above which unstable modes are predicted to contribute significantly to the fluxes, and taking a typical $R / L_{T}$ value of 9 , we obtain: $s=90 \frac{\rho_{s}}{R}$. For mid-sized tokamaks, a typical value of $\frac{\rho_{s}}{R}$ is 0.001 , thus a typical $s$ for which the locality assumption fails is 0.1 , higher for smaller tokamaks and lower for larger tokamaks.

\section{Appendix C: Analytical fluid eigenmode assumption}

In this appendix we show, in comparisons with linear-GENE, that the analytical fluid Gaussian eigenmode approximation made in QuaLikiz, and the strong ballooning representation inherent therein, are valid for $|s|>0.1$. 
The scale separation of the microinstabilities, where $k_{\|} \ll k_{\perp}$, is captured in the eikonal representation for the electrostatic potential perturbation: $\Phi_{n}(r, \theta, \varphi)=e^{-i n(\varphi-q(r) \theta)} \phi_{n}(r, \theta)$, where $\phi_{n}$ has slow varying $\theta$-dependence along the field line. To first order $q(r) \approx q\left(r_{0}\right)+\left.x q^{\prime}\right|_{r_{0}}$, with $r_{0}$ corresponding to the resonating surface where $q\left(r_{0}\right)=m_{0} / n$, and $x \equiv r-r_{0}$, the radial distance from the resonating surface. The poloidal harmonic of $\Phi_{n}$ is thus:

$$
\tilde{\phi}_{m n}(r)=\frac{1}{2 \pi} \int_{-\pi}^{\pi} \phi_{n}(\theta) e^{i \theta\left(\frac{x}{d}-\nu\right)} d \theta
$$

With $\nu=m-m_{0}, d=1 / n q^{\prime}$, and where we have assumed radial dependence only through $q(r)$. In the QuaLiKiz model, $\tilde{\phi}(r)$ is not solved, but prescribed from the fluid limit solution, which is a Gaussian: $\tilde{\phi}(r)=\phi_{0} e^{-r^{2} / 2 w^{2}}$, with $w$ the Gaussian width ${ }^{8}$. The fluid limit solution is outlined in appendix A. If $\phi_{n}(\theta)$ vanishes sufficiently quickly at $\pm \pi$, then equation $\mathrm{C} 1$ describes a Fourier transform. $\phi_{n}(\theta)$ is then the conjugate of $\phi_{n}(r)$, with $\theta=k_{r} d$. However, for low shear and long wavelengths, this assumption may fail since the prescribed $\phi\left(k_{r}\right)$ solution may not be compatible with $\phi(\theta)$ periodicity since then $\phi(\theta)$ may not necessarily vanish at $\pm \pi$. Indeed, it is known that as $s \rightarrow 0$ the full ballooning representation solution of $\phi(\theta)$ tends to a Mathieu function ${ }^{38}$, which is not localized is the vicinity $\theta=0$. Therefore we expect that below a particular value of $s$, the QuaLiKiz Gaussian eigenmode assumption must break down.

The validity of the QuaLiKiz assumption that the eigenmode is a Gaussian and vanishing at $\pm \pi$, i.e. $\phi_{n}(\theta= \pm \pi) \rightarrow 0$, is examined versus the solution of $\phi_{n}(\theta)$ from linear GENE, displayed in the ballooning representation ${ }^{39}$. The QuaLiKiz/GENE comparison is seen in figure 16 for the GA standard case at both $s=0.1$ and $s=1$. We examine the cases with the transport relevant $k_{y}=0.1,0,15,0.2$. The GENE calculated modes tend to be more extended in the ballooning angle for the combination of low s and low $k_{y}$. The QuaLiKiz assumption holds for all cases apart from $s=0.1, k_{y}=0.1$. However, since the peak of the non-linear flux spectrum for the GA-standard $s=0.1$ case falls between $k_{y}=0.15-0.2$, the linear calculations for $k_{y}=0.1$ are less critical in the low shear case (see section IV C). We can conclude that the lowest order ballooning representation assumption made in QuaLiKiz holds down to $s=0.1$. 

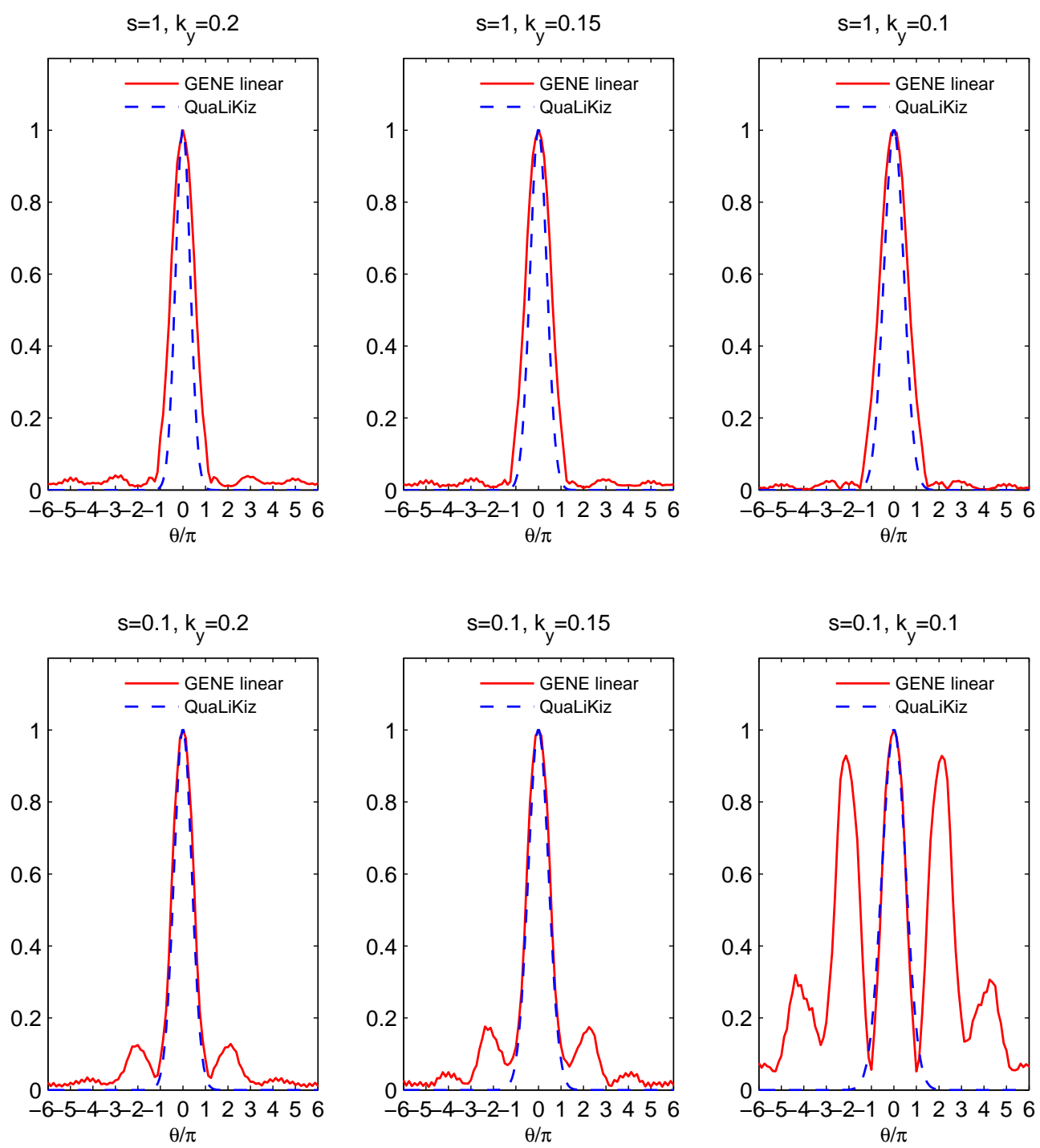

FIG. 16. Calculated GeNE linear eigenfunctions compared with the QuaLiKiz strong ballooning assumption for the mode envelope, for $\mathrm{s}=1$ (upper panels) and $\mathrm{s}=0.1$ (lower panels) GASTD case parameters

\section{Appendix D: Improvement in trapped particle functional}

QuaLiKiz has been modified to improve the linear growth rate predictions for TEM dominated regimes by including additional terms for the bounce frequency and bounce average treatment in the trapped particle functional. The dispersion relation arising from the linearized Vlasov equation combined with the electroneutrality constraint condition can 
formally be written as $^{40}$ :

$$
\sum_{s} \mathcal{L}_{s, t}+\mathcal{L}_{s, p}=0
$$

$\mathcal{L}_{s, t}$ and $\mathcal{L}_{s, p}$ are trapped and passing particle functionals for each species, given as:

$$
\begin{aligned}
\mathcal{L}_{s, p} & =\left\langle\iint_{-\infty}^{+\infty} \frac{\mathrm{d} k \mathrm{~d} x}{\pi} J_{0}^{2}\left(k_{\perp} \rho_{s}\right)|\tilde{\phi}(x, k)|^{2} \frac{\omega-n \omega_{s}^{*}}{\omega-n \omega_{d}^{p}-k_{\|} v_{\|}+\imath 0^{+}}\right\rangle_{p} \\
\mathcal{L}_{s, t} & =\left\langle\sum_{m} \int_{-\infty}^{+\infty} \frac{\mathrm{d} k_{r}}{2 \pi} J_{0}^{2}\left(k_{\perp} \rho_{s}\right) J_{m}^{2}\left(k_{r} \delta_{s}\right)\left|\tilde{\phi}\left(k_{r}\right)\right|^{2} \frac{\omega-n \omega_{s}^{*}}{\omega-n \omega_{d}^{t}-m \omega_{b}+\imath 0^{+}}\right\rangle_{t}
\end{aligned}
$$

The integrations $\langle\cdots\rangle_{t, p}$ are defined as:

$$
\begin{aligned}
\langle\ldots\rangle_{t} & =\int_{0}^{+\infty} \frac{2}{\sqrt{\pi}} \sqrt{\mathcal{E}} \mathrm{d} \mathcal{E} \int_{\lambda_{c}}^{1} \frac{\mathrm{d} \lambda}{4 \bar{\omega}_{b}}=f_{t} \int_{0}^{+\infty} \frac{2}{\sqrt{\pi}} \sqrt{\mathcal{E}} \mathrm{d} \mathcal{E} \int_{0}^{1} K(\kappa) \kappa \mathrm{d} \kappa \\
\langle\ldots\rangle_{p} & =\int_{0}^{+\infty} \frac{2}{\sqrt{\pi}} \sqrt{\mathcal{E}} \mathrm{d} \mathcal{E} \int_{0}^{\lambda_{c}} \frac{\mathrm{d} \lambda}{4 \bar{\omega}_{b}}
\end{aligned}
$$

$\omega_{d}^{t}$ is the trapped particle vertical drift frequency, taken as $n \omega_{d}^{t}=-\frac{k_{\theta} T_{s}}{e_{s} B} f(\kappa)$ where $f(\kappa)=2 \frac{E(\kappa)}{K(\kappa)}-1+4 s\left(\kappa^{2}-1+\frac{E(\kappa)}{K(\kappa)}\right)$, and $K(\kappa)$ and $E(\kappa)$ are first and second order complete elliptic integrals. $n \omega_{d}^{p}$ is the passing particle vertical drift frequency: $n \omega_{d}^{p}=$ $-\frac{k_{\theta} T_{s}}{e_{s} B}(\cos \theta+(s \theta-\alpha \sin \theta) \sin \theta) . \quad \omega_{s}^{*}$ is the diamagnetic frequency for the $s$ species, $\omega_{b}= \pm \frac{V_{T s}}{q R} \sqrt{\mathcal{E}} \bar{\omega}_{b}$ is the bounce frequency, $m$ is the poloidal wave number, $J_{m}$ is the $\mathrm{m}$ order Bessel function and the poloidal harmonic $\tilde{\phi}$ is the eigenfunction (taken from the analytical fluid limit). Finally $\bar{\omega}_{b}$ measures the effects of the variation of the pitch angle $\lambda$ around a poloidal revolution.

In the previous version of QuaLiKiz, only the $m=0$ term in Eq.D3 was implemented. In most cases the $m \neq 0$ terms are negligible contributors to the resonance. However, in some cases - such as at high $|s|$ or for low $T_{i}$ gradients - the influence of the bounce frequency $\omega_{b}$ on the resonance can be significant. Therefore the $m=1$ term has been incorporated in QuaLiKiz.

\section{Appendix E: Improvement of the mode width calculation at high $k_{y}$}

In this appendix, the modification of the QuaLiKiz mode width calculation for high $k_{y}$ ETG modes is reviewed. The electrostatic potential eigenmode is not self-consistently calculated in QuaLiKiz, but rather prescribed from a fluid limit analytical calculation as seen 
in equation A1. In this limit, the eigenfrequencies of the unstable modes are considered much greater than the drift and diamagnetic frequencies. Furthermore, $k_{y}<1$ when assessing the ordering of the various terms. The dispersion relation is linearized under these assumptions, where Bessel functions containing ion scales are expanded to second order, and Bessel functions containing electron scales expanded to zeroth order. $\phi$ is then the Gaussian solution of the resulting second order differential equation. However, for high wave numbers corresponding to ETG modes - typically $k_{y}>2$ - the assumptions regarding the linearization of the Bessel functions in the dispersion relation are not satisfied. In this case, we can assume adiabatic ions (equate Bessel functions containing ion scales to zero), and assuming $k_{\theta} \rho_{e} \ll 1$ expand the Bessel functions containing electron scales to second order. In these conditions the linearized gyrokinetic dispersion relation reads:

$$
\left[\frac{Z_{e f f}}{\tau}+\frac{n \omega_{n e}^{*}}{\omega}-\left(1-\frac{n \omega_{p e}^{*}}{\omega}\right)-\frac{n \omega_{d}}{\omega}+f_{p}\left(1-\frac{n \omega_{p e}^{*}}{\omega}\right)\left(\frac{k_{\perp}^{2} d_{e f f}^{2}}{2}-\frac{n \omega_{d}}{\omega}(s-\alpha-0.5)-\frac{k_{\|}^{2} v_{T_{e}}^{2}}{2 \omega^{2}}\right)\right] \tilde{\phi}=0
$$

where $d_{e f f}=\frac{f_{t}}{f_{p}} \delta_{e}+\rho_{e}$ in this case. This equation is similar to equation A1, and as in the previous case the solution for $\phi$ is a Gaussian whose width $w$ is given by:

$$
w^{4}=-\frac{\omega^{2}\left(d_{e f f}^{2}-2 d^{2}(s-\alpha-0.5)\right)}{\left(\frac{k_{\theta} s}{q R} v_{T_{e}}\right)^{2}}
$$

where the ratio $\frac{n \omega_{d}}{\omega}$ is considered equal to 1 . We obtain a quadratic equation for $\omega$ :

$\Omega^{2}\left(\frac{Z_{e f f}}{\tau}+f_{p} \frac{k_{\theta}^{2} d_{e f f}^{2}}{2}\right)+\Omega\left(\omega_{n e}^{*}-\omega_{d}-f_{p} \frac{k_{\theta}^{2} d_{e f f}^{2}}{2} \omega_{p e}^{*}+\imath f_{p} \frac{k_{\theta} s V_{T_{e}} d_{e f f}}{2 n q R}\right)+\omega_{p e}^{*} \omega_{d}-\imath f_{p} \frac{k_{\theta} s v_{T_{e}} d_{e f f}}{2 n q R} \omega_{p e}^{*}=0$

In the interchange limit the solution is $\gamma^{2}=\frac{n \omega_{p e}^{*} n \omega_{d}}{\frac{Z_{e f f}}{\tau}}$. In the slab limit the solution is $\gamma^{2}=\frac{k_{\theta} s v_{T_{e}} d_{e f f} n \omega_{p e}^{*}}{2 q R \frac{Z_{e f f}}{\tau}}$

The new eigenfunction at high $k_{y}$ has been validated by linear GENE, as seen in figure 17. The new mode width is much closer to the GENE calculated eigenmode at high $k_{y}$. A flag has been implemented in QuaLiKiz to switch between the low $k_{y}$ and high $k_{y}$ versions of the linearized dispersion relation for the mode width calculation, based on an assessment of the degree of adiabicity of the passing electrons. This switch is typically triggered within the range $k_{y} \sim 1-2$. The normalized linear growth rates from both GENE and QuaLiKiz, as a function of $k_{y}$, can be seen in figure 18. The scan was carried out for $R / L_{T_{i}}=0$ 


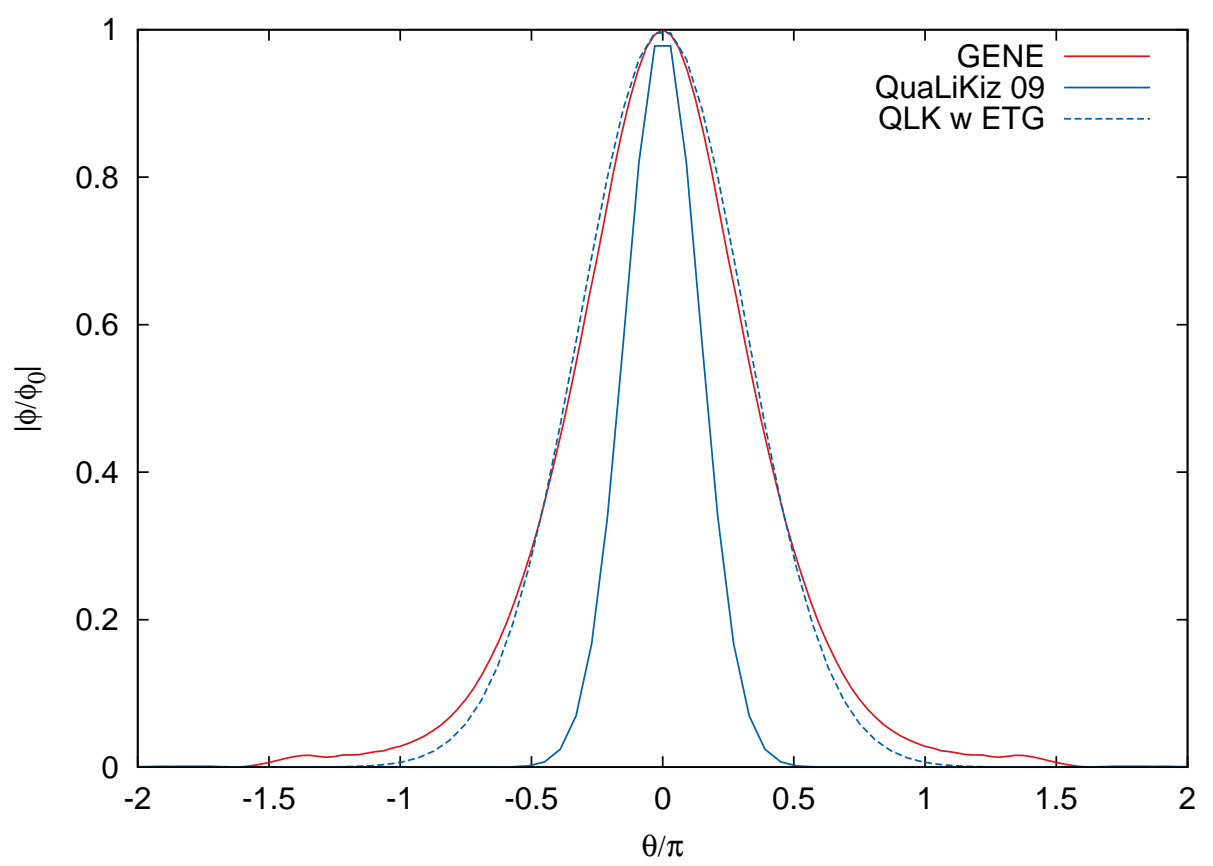

FIG. 17. Electrostatic potentials calculated by Gene and QuaLiKiz for $k_{y}=30$

with the remaining parameters corresponding to the GA-standard case. At high $k_{y}$, the agreement between linear growth rates of QuaLiKiz and Gene is clearly improved. This could be of importance for the accurate modelling of ETG modes, which may, for example, be responsible for residual electron transport in transport barriers.

\section{Appendix F: Improvement of the mode width calculation around $s=0.5$}

QuaLiKiz was also modified to correct an inaccuracy of the model in the region $s \sim 0.5$, linked to the mode width calculation. The expression for the linearized gyrokinetic dispersion relation is repeated here:

$$
\left[1+\frac{f_{t}}{f_{p}}\left(1+\frac{\tau}{\bar{Z}}\right) \frac{n \omega_{p e}^{*} n \omega_{d}^{t}}{\omega^{2}}-\frac{n \omega_{n e}^{*}}{\omega}+\left(1+\frac{\tau}{\bar{Z}} \frac{n \omega_{p e}^{*}}{\omega}\right)\left(\frac{n \omega_{d}^{p}}{\omega}-\frac{k_{\|}^{2} c_{e f f}^{2}}{2 \omega^{2}}+\frac{k_{\perp}^{2} d_{e f f}^{2}}{2}\right)\right] \tilde{\phi}=0
$$

where $f_{t}$ and $f_{p}$ are the fraction of trapped and passing particles respectively, $d_{e f f}$ is an effective banana width which takes into account finite Larmor radius (FLR) and finite banana width (FBW) effects: $d_{\text {eff }}^{2}=\rho_{\text {eff }}^{2}+f_{t} / f_{p} \delta_{\text {eff }}^{2}$ where $\rho_{\text {eff }}^{2}=\frac{4 T_{e} m_{p}}{e^{2} B^{2}}$ is the effective 


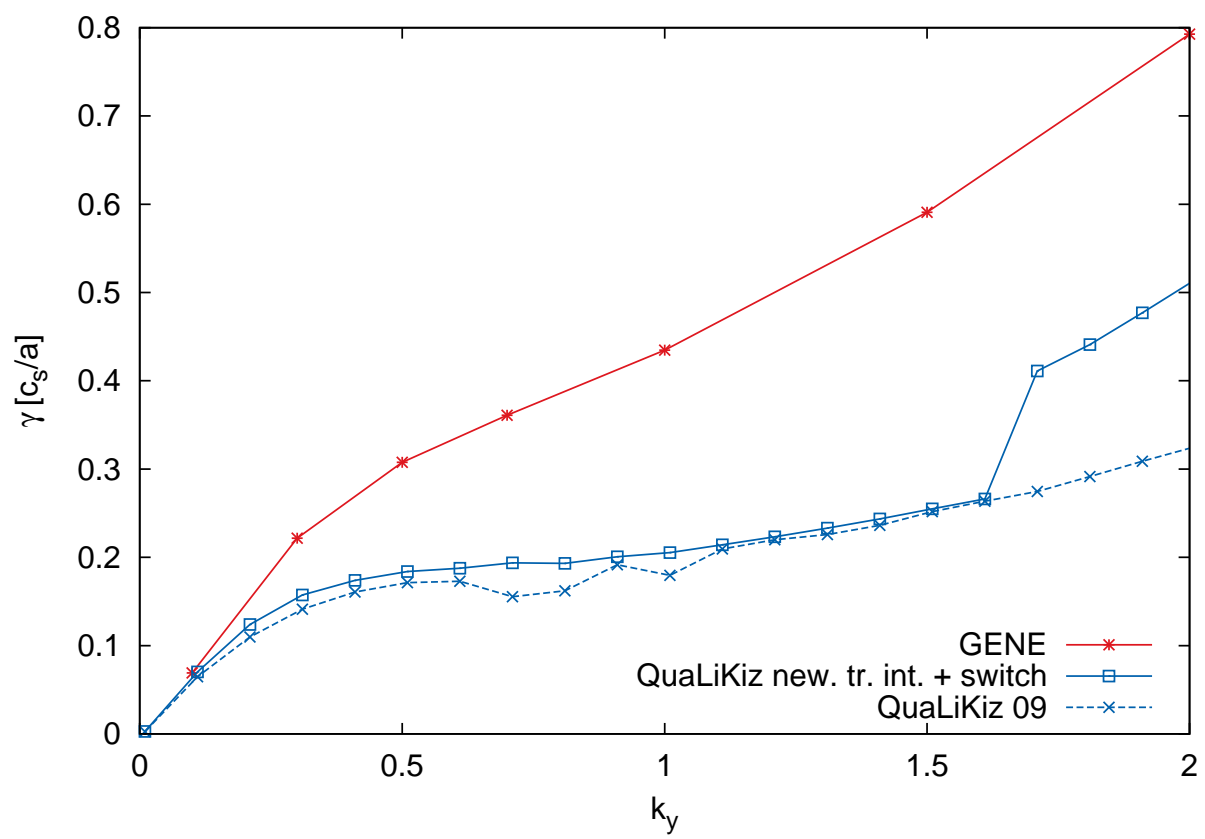

FIG. 18. Linear growth rates calculated by GENE and QuaLiKiz for the TEM scenario

Larmor radius and $\delta_{\text {eff }}^{2}=\frac{q^{2}}{2 \epsilon} \rho_{\text {eff }}^{2}$ is the effective banana width. $\quad c_{\text {eff }}=\sqrt{\frac{T_{e}}{m_{p}}}$ is the effective thermal velocity and $\frac{\tau}{Z}=\sum_{i} \frac{\nabla_{r} P_{i}}{\nabla_{r} P_{e}}$ is the ratio of the pressure gradients between electrons and ions. The diamagnetic frequency has been split into two terms: one due to the density gradients $-n \omega_{n s}^{*}=-\frac{k_{\theta} T_{s}}{e_{s} B} \frac{\mathrm{d}}{\mathrm{d} r} \log n_{s}$ - and one due to the pressure gradients $n \omega_{p s}^{*}=-\frac{k_{\theta} T_{s}}{e_{s} B}\left(\frac{\mathrm{d}}{\mathrm{d} r} \log n_{s}+\frac{\mathrm{d}}{\mathrm{d} r} \log T_{s}\right)$. Note that equation $\mathrm{F} 1$ is identical to equation $\mathrm{A} 1$ apart from the separation of $n \omega_{d}$ into distinct trapped and passing particle expressions. This is due to the fact that $n \omega_{d}^{p}$ and $n \omega_{d}^{t}$ are identical only to $0^{\text {th }}$ order in $\theta$. The passing particle expression is:

$$
n \omega_{d}^{p}=-\frac{k_{\theta} T_{s}}{e_{s} B R}(\cos \theta+(s \theta-\alpha \sin \theta) \sin \theta)
$$

whereas the expression of $n \omega_{d}^{t}$ in the deeply trapped limit is: $n \omega_{d}^{t}=-\frac{k_{\theta} T_{s}}{e_{s} B R}=n \omega_{d}$. When expanding $\omega_{d}^{p}$ to second order in $\theta$, we obtain:

$$
n \omega_{d}^{p}=-\frac{k_{\theta} T_{s}}{e_{s} B R}\left(1+\theta^{2}(s-\alpha-0.5)\right)
$$

Equation $\mathrm{F} 1$ is the same as equation A1 apart from the following term, proportional to $s$ : $\left(1+\frac{\tau}{Z} \frac{n \omega_{\omega e}^{*}}{\omega}\right) \frac{n \omega_{d}}{\omega}\left(k_{r} d\right)^{2}(s-\alpha-0.5)$. This leads to a modified expression for $w$ compared to 
the expression presented in appendix $\mathrm{A}^{9}$ :

$$
w^{4}=-\frac{\left(d_{e f f}^{2}+2 \frac{f_{t}}{f_{p}} d^{2}(s-\alpha-0.5)\right) \omega^{2}}{\left(\frac{k_{\theta} s}{q R} c_{e f f}\right)^{2}}
$$

where the ratio $\frac{n \omega_{d}}{\omega}$ has been taken equal to 0.5 .

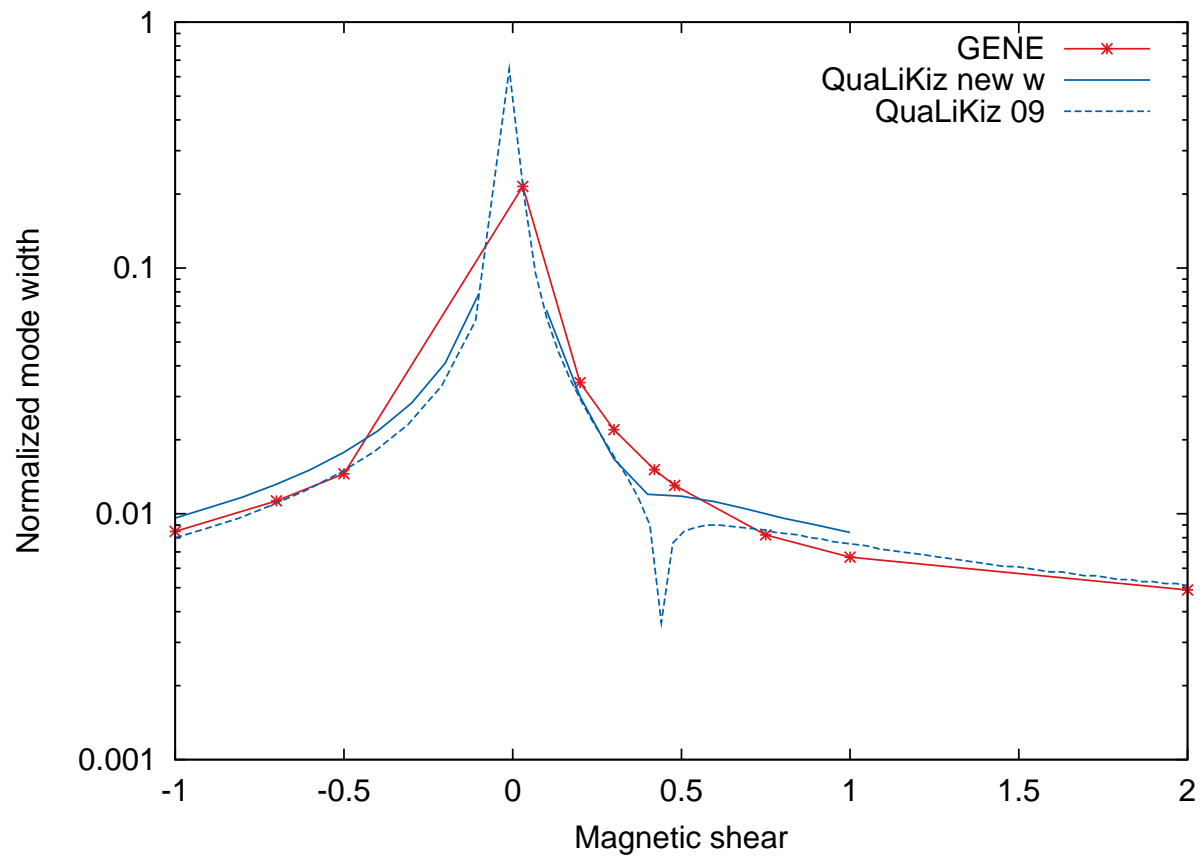

FIG. 19. Mode width (normalized to the minor radius) calculated by GENE and QuaLiKiz for $k_{y}=0.3$

With this definition for $w$, the divergence issue mentioned in $^{8}$ around $s=0$ is resolved. However, there is still an irregularity around $s=0.5$ as can be seen in the dashed curve in figure 19. This effect is shown for $k_{y}=0.3$ as an example, but this irregularity is present for all wave numbers, although somewhat smoother at greater $k_{y}$. This irregularity stems from the second order expansion the vertical drift frequency $n \omega_{d}^{p}$.

In the current version of QuaLiKiz, $n \omega_{d}^{p}$ was expanded to sixth order in $\theta$. The higher order expansion removes the irregularity at $s=0.5$ as seen in the solid curve in figure 19 . The new expression for $n \omega_{d}^{p}$ is now:

$$
n \omega_{d}^{p}=n \omega_{d}\left[1+\theta^{2}(s-\alpha-0.5)-\frac{\theta^{4}}{6}(s-2 \alpha-0.25)+\frac{\theta^{6}}{120}\left(s-\frac{16}{3} \alpha-\frac{1}{6}\right)\right]
$$


This leads to a new recursive expression for $w$ :

$w^{4}=-\left(\frac{\omega d_{e f f}}{\frac{k_{\theta} s}{q R} c_{e f f}}\right)^{2}\left[1+2 \frac{f_{t} d^{2}}{f_{p} d_{e f f}^{2}}\left(s-\alpha-0.5+\frac{d^{2}}{w^{2}}(s-2 \alpha-0.25)+\frac{5}{80} \frac{d^{4}}{w^{4}}(6 s-32 \alpha-1)\right)\right]$

All improvements implemented in QuaLiKiz are summarized in figure 20. A scan in mag-

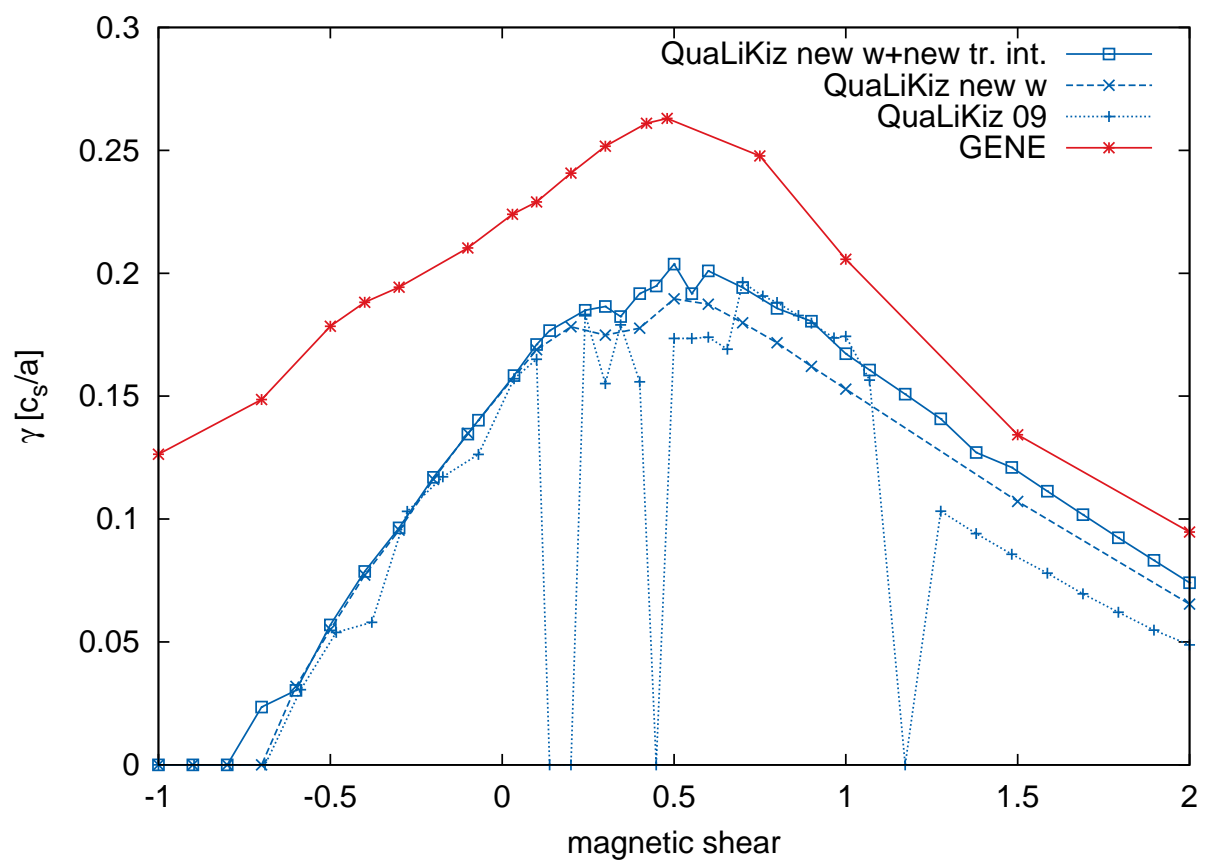

FIG. 20. Linear growth rates of GENE and QuaLiKiz for the GA-standard case (apart from $r / a=0.25)$ at $k_{y}=0.3$.

netic shear is presented with the different versions of QuaLiKiz for GA-standard case parameters, but at reduced radius: $r / a=0.25$. The dotted curve represents the version of QuaLiKiz used $\mathrm{in}^{4}$, the dashed curve illustrates the improvements brought by the new developments of the mode width. The solid curve is the result when the new term for trapped particles is added. Figure 20 was chosen to be plotted for $k_{y}=0.3$, a typical wavenumber contributing significantly to the transport flux. However, the improvements due to the new high- $k_{y}$ calculation are thus not evident from this graph, but those improvements are evident in figure 18 . 


\section{REFERENCES}

${ }^{1}$ C. Bourdelle, X. Garbet, F. Imbeaux, A. Casati, N. Dubuit., R. Guirlet, and T. Parisot, Phys. Plasmas 14, 112501 (2007)

${ }^{2}$ J. Candy and R. Waltz, J. Comp. Phys. 186, 545 (2003)

${ }^{3}$ J. Candy and R. Waltz, Phys. Rev. Lett. 91, 045001 (2003)

${ }^{4}$ A. Casati, C. Bourdelle, X. Garbet, F. Imbeaux, J. Candy, F. Clairet, G. Dif-Pradalier, G. Falchetto, T. Gerbaud, V. Grandgirard, Ö. Gürcan, P. Hennequin, J. Kinsey, M. Ottaviani, R. Sabot, Y. Sarazin, L. Vermare, and R. Waltz, Nucl. Fusion 49, 085012 (2009) ${ }^{5}$ P. Mantica, C. Angioni, C. Challis, G. Colyer, L. Frassinetti, N. Hawkes, T. Johnson, M. Tsalas, P. C. deVries, J. Weiland, B. Baiocchi, M. Beurskens, A. Figueiredo, C. Giroud, J. Hobirk, E. Joffrin, E. Lerche, V. Naulin, A. Peeters, A. Salmi, C. Sozzi, D. Strintzi, G. Staebler, T. Tala, D. V. Eester, and T. Versloot, Phys. Rev. Lett. 104, 135004 (2011) ${ }^{6}$ F. Porcelli, D. Boucher, and M. Rosenbluth, Plasma Phys. Control. Fusion 38, 2163 (1996) ${ }^{7}$ F. Jenko, W. Dorland, M. Kotschenreuther, and B. Rogers, Phys. Plasmas 7, 1904, and http://gene.rzg.mpg.de (2000)

${ }^{8}$ C. Bourdelle, X. Garbet, G. Hoang, J. Ongena, and R. Budny, Nucl. Fusion 42, 892 (2002) ${ }^{9}$ M. Romanelli, G. Regnoli, and C. Bourdelle, Phys. Plasmas 14, 082305 (2007)

${ }^{10}$ A. Casati, Ph.D. thesis, Universite de Provence (Aix-Marseille I) (2009)

${ }^{11}$ A. Casati, T. Gerbaud, P. Hennequin, C. Bourdelle, J. Candy, F. Clairet, X. Garbet, V. Grandgirard, Ö. Gürcan, S. Heuraux, G. Hoang, C. Honoré, F. Imbeaux, R. Sabot, Y. Sarazin, L. Vermare, and R. E.Waltz, Phys. Rev. Lett. 102, 165005 (2009) ${ }^{12}$ F. Jenko, T. Dannert, and C. Angioni, Plasma Phys. Control. Fusion 47, B195 (2005) ${ }^{13}$ J. Kinsey, R. Waltz, and J. Candy, Phys. Plasmas 13, 022305 (2006) ${ }^{14}$ C. Bourdelle, Ph.D. thesis, Universite Joseph Fourier-Grenoble 1 (2000) ${ }^{15}$ S. Guo and F. Romanelli, Phys. Fluids B 5, 520 (1993)

${ }^{16}$ M. Romanelli, C. Bourdelle, and W. Dorland, Phys. Plasmas 11, 3845 (2004)

${ }^{17}$ G. Hammett, W. Dorland, and F. Perkins, Phys. Fluids B 4, 2052 (1993)

${ }^{18}$ T. Hahm and W. Tang, Phys. Fluids B 1, 1185 (1989)

${ }^{19}$ C. Fourment, G. Hoang, L.-G. Eriksson, X. Garbet, X. Litaudon, and G. Tresset, Plasma Phys. Control. Fusion 45, 233 (2003)

${ }^{20}$ A. Casati, C. Bourdelle, X. Garbet, and F. Imbeaux, Phys. Plasmas 15, 042310 (2008) 
${ }^{21}$ F. Jenko, W. Dorland, and G. Hammett, Phys. Plasmas 8, 4096 (2001)

${ }^{22}$ F. Romanelli, Liu. Chen, and S. Briguglio, Phys. Fluids B 3, 2496 (1991)

${ }^{23}$ R. Kubo, J. Math. Phys. 4, 174 (1963)

${ }^{24}$ J. Krommes, Physics Reports 360, 1 (2002)

${ }^{25}$ D. Escande and F. Sattin, Phys. Rev. Lett. 99, 185005 (2007)

${ }^{26}$ Z. Lin, I. Holod, L. Chen, P. Diamond, T. Hahm, and S. Ethier, Phys. Rev. Lett. 99, $265003(2008)$

${ }^{27}$ T. Dannert and F. Jenko, Phys. Plasmas 12, 072309 (2005)

${ }^{28}$ P. Diamond, S.-I. Ito, K. Itoh, and T. Hahm, Plasma Phys. Control. Fusion 47, R31 (2005)

${ }^{29}$ R. Waltz, G. Staebler, W. Dorland, G. Hammett, M. Kotschenreuther, and J. Konings, Phys. Plasmas 4, 2482 (1997)

${ }^{30}$ J. Kinsey, G. Staebler, and R. Waltz, Phys. Plasmas 12, 052503 (2005)

${ }^{31}$ J. Kinsey, G. Staebler, and R. Waltz, Phys. Plasmas 15, 055908 (2008)

${ }^{32}$ T. Hahm, M. Beer, Z. Lin, G. Hammett, W. Lee, and W. Tang, Phys. Plasmas 6, 922 (1999)

${ }^{33}$ Li. Jiquan and Y. Kishimoto, Phys. Plasmas 12, 054505 (2005)

${ }^{34}$ F. Hinton and G. Staebler, Phys. Fluids B 5, 1281 (1993)

${ }^{35}$ R. Waltz, A. Casati, and G. Staebler, Phys. Plasmas 16, 072303 (2009)

${ }^{36}$ D. Hatch, P. Terry, W. Nevins, and W. Dorland, Phys. Plasmas 16, 022311 (2009)

${ }^{37}$ D. Hatch, P. Terry, F. Jenko, F. Merz, and W. Nevins, Phys. Rev. Lett. 106, 115003 (2011)

${ }^{38}$ J. Candy, R. Waltz, and M. Rosenbluth, Phys. Plasmas 11, 1879 (2004)

${ }^{39}$ J. Connor, R. Hastie., and J. Taylor, Phys. Rev. Lett. 40, 396 (1978)

${ }^{40}$ X. Garbet, L. Laurent, F. Mourgues, and J. P. Samain, J. Comp. Phys. 87, 249 (1990) 